\title{
Abundances from integrated spectra of 47 Tucanae (NGC 104)
}

\author{
C. Rennó, ${ }^{1}$ B. Barbuy ${ }^{\oplus},{ }^{1 \star}$ T. C. Moura ${ }^{\oplus \star}$ and M. Trevisan $^{\circledR 2 \star}$ \\ ${ }^{1}$ IAG, Universidade de São Paulo, Rua do Matão 1226, Cidade Universitária, São Paulo 05508-900, Brazil \\ ${ }^{2}$ Departamento de Astronomia, Universidade Federal do Rio Grande do Sul, CP 15051, Porto Alegre 91501-970, Brazil
}

Accepted 2020 September 2. Received 2020 September 2; in original form 2020 July 15

\begin{abstract}
47 Tucanae is among the most studied and observed globular clusters, given its proximity. The aim of this work is to study in detail the integrated spectrum of 47 Tucanae, as a template, in order to have a list of reliable lines that are validated for a moderate spectral resolution case. The spectrum of 47 Tucanae is reproduced by computing synthetic spectra, taking into account individual element abundances. The results are compared with other methods. We reproduce the integrated spectrum in the range 4500-9000 $\AA$ and derive abundances from individual lines of $\mathrm{Na}, \mathrm{Mg}, \mathrm{Al}, \mathrm{Si}, \mathrm{Ca}, \mathrm{Ti}, \mathrm{Ba}$, and Eu. We report a list of lines that are suitable for abundance derivation. Adopting these abundances, we are able to fit the well-known triplets of $\mathrm{Mg}$ I and Ca II. Finally, the effect of multiple stellar populations through enhanced $\mathrm{Na}$ abundances are tested. Element abundances derived are compatible with previous literature abundances, from different methods. The method applied here to build integrated synthetic spectra can be used to derive abundances from observed integrated spectra of distant and faint clusters, that are usually observed at low or moderate spectral resolution.
\end{abstract}

Key words: globular clusters: individual: (47 Tucanae).

\section{INTRODUCTION}

47 Tucanae (hereafter 47 Tuc) is among the closest globular clusters in the Galaxy at $d_{\odot} \sim 4.5 \mathrm{kpc}$, together with M4 (NGC 6121) at $2.2 \mathrm{kpc}$, and NGC 6397 at $2.3 \mathrm{kpc}$. Its apparent magnitude of $M_{\mathrm{V}}=$ 3.95 makes it the second brightest cluster in the sky, only behind $\omega$ Centauri (NGC 5139) (Harris 1996, edition 2010). For these reasons this cluster has been extensively studied since the early work by Shapley (1918). It is an interesting cluster because its location, kinematics, and metallicity were interpreted in different terms along the years. For example, it was classified by Armandroff (1989) as a disc cluster. Bica, Ortolani \& Barbuy (2016) included 47 Tuc in a list of clusters intruders to the bulge, characterized by $[\mathrm{Fe} / \mathrm{H}]>-1.0$ and $d_{\circ}>4.5 \mathrm{kpc}$. Barbuy, Chiappini \& Gerhard (2018b) and Ortolani et al. (1995) have considered 47 Tuc as an inner halo cluster. More recently, Pérez-Villegas et al. (2020) computed its orbits based on proper motions from the Gaia collaboration (2018a). They computed minimum and maximum distances to the Galactic Centre of 7.2 and $9.4 \mathrm{kpc}$, a height relative to the Galactic plane of $4.5 \mathrm{kpc}$, and an eccentricity of 0.13 , for a bar rotation speed of $40 \mathrm{~km} \mathrm{~s}^{-1} \mathrm{kpc}^{-1}$, and concluded that this is an inner halo cluster.

47 Tuc is located at $\alpha(\mathrm{J} 2000) 00^{\mathrm{h}} 24^{\mathrm{m}} 05^{\mathrm{s}} 359, \delta(\mathrm{J} 2000)=$ $-72^{\circ} 04^{\prime} 53^{\prime \prime} 20, l=305.89, b=-44.89$, with distances in the literature ranging from $4.5 \pm 0.45$ (Harris 1996, edition of 2010; Bica et al. 2006; Baumgardt et al. 2019), to $5.10 \mathrm{kpc}$ from the Gaia Collaboration (2018b) with the distance based on the inverse of its parallax (1/plx; see Pérez-Villegas et al. 2020). A reddening of $E(B$

^E-mail: b.barbuy@iag.usp.br (BB); tatiana.mourabastos@gmail.com (TCM); marina.trevisan@ufrgs.br (MT)
$-V)=0.03$ (Brogaard et al. 2017), and an absolute magnitude $M_{\mathrm{V}}=$ -9.42 (Vandenberg et al. 2013) are assigned to 47 Tuc.

Spectroscopic analyses of individual stars of 47 Tuc are available since the late 1970s (e.g. Dickens, Bell \& Gustafsson 1979; Norris \& Cottrell 1979; Pilachowski, Canterna \& Wallerstein 1980). Highresolution spectroscopy (HRS) of individual red giant branch (RGB) stars with $8 \mathrm{~m}$ class telescopes was carried by several groups (AlvesBrito et al. 2005; Koch \& McWilliam 2008; Carretta et al. 2009; Cordero et al. 2014; Thygesen et al. 2014), resulting in metallicities in the range $-0.79<[\mathrm{Fe} / \mathrm{H}]<-0.66$.

Integrated spectra of globular clusters are particularly useful as a basis for the study of composite spectra of galaxies, as first carried out by Bica, Alloin \& Schmidt (1990), and Burstein et al. (1984), who indicated that studying the stellar populations of globular clusters is a crucial step for understanding better the stellar populations in galaxies, since clusters are a less complex structure. This remains valid even though there is now ample evidence that GCs contain multiple populations of stars (e.g. Milone et al. 2017). Only recently, higher resolution integrated spectra were observed in order to be analysed with the same quality as earlier for individual stars. Such studies have been carried out both by deriving chemical abundances using equivalent widths (hereafter EWs; McWilliam \& Bernstein 2008; Colucci, Bernstein \& McWilliam 2017) or full spectral fitting (Larsen, Brodie \& Strader 2017; Conroy et al. 2018), besides other methods (Graves \& Schiavon 2008; Usher et al. 2019). A compilation on chemical abundances of globular clusters was presented by Roediger et al. (2014).

The aim of this work is to derive abundances through integrated synthetic spectra compared with the recently released integrated spectra of 47 Tuc obtained with the integral field unit WideField spectrograph (WiFes) on the Australian National University $2.3 \mathrm{~m}$ telescope and presented by Usher et al. (2017), contained in 
Table 1. Literature metallicities and abundances. The data are divided by objects and resolution of the spectra used in the analysis, where they are divided by high resolution (HRS), integrated, and medium resolution (MRS) spectra. The last value in the last box is from Roediger et al. (2014), that made a compilation of the results from studies prior of this publication on this topic.

\begin{tabular}{|c|c|c|c|c|c|c|c|c|c|c|c|c|}
\hline Cluster & {$[\mathrm{Fe} / \mathrm{H}]$} & [O/Fe] & {$[\mathrm{Na} / \mathrm{Fe}]$} & {$[\mathrm{Al} / \mathrm{Fe}]$} & {$[\mathrm{Mg} / \mathrm{Fe}]$} & {$[\mathrm{Si} / \mathrm{Fe}]$} & {$[\mathrm{Ca} / \mathrm{Fe}]$} & [Ti/Fe] & {$[\mathrm{Ba} / \mathrm{Fe}]$} & {$[\mathrm{Eu} / \mathrm{Fe}]$} & Reference & Method \\
\hline NGC 104 & -0.76 & 0.60 & 0.20 & 0.45 & 0.45 & 0.40 & 0.34 & 0.36 & - & - & 1 & HRS \\
\hline NGC 104 & -0.79 & 0.21 & 0.34 & 0.38 & - & 0.28 & 0.28 & 0.34 & - & 0.44 & 3 & HRS \\
\hline NGC 104 & -0.78 & 0.30 & 0.21 & 0.21 & 0.44 & 0.32 & 0.24 & 0.33 & 0.25 & 0.32 & 4 & HRS \\
\hline NGC 104 & -0.77 & 0.25 & 0.53 & 0.52 & 0.52 & 0.40 & - & - & - & - & 5 & HRS \\
\hline NGC 104 & -0.72 & - & - & - & - & - & - & - & - & - & 6 & HRS \\
\hline \multicolumn{13}{|c|}{ Asymptotic giant branch individual stars } \\
\hline NGC 104 & -0.60 & - & 0.65 & - & - & - & - & - & - & 0.14 & 8 & HRS \\
\hline NGC 104 & -0.83 (Fe II) & - & - & - & - & - & - & - & - & - & 9 & HRS \\
\hline NGC 104 & $-0.94(\mathrm{Fe} I)$ & - & - & - & - & - & - & - & - & - & 10 & HRS \\
\hline \multicolumn{13}{|c|}{ Integrated spectra } \\
\hline NGC 104 & -0.84 & - & - & - & 0.42 & 0.27 & 0.23 & 0.40 & - & - & 15 & ISFF \\
\hline \multicolumn{13}{|c|}{ Compilation } \\
\hline NGC 104 & -0.72 & 0.24 & 0.35 & - & 0.41 & 0.31 & 0.17 & 0.28 & - & - & 16 & compilation \\
\hline
\end{tabular}

References: 1 - Koch \& McWilliam (2008) with data from Magellan Inamori Kyocera Echelle high-resolution spectrograph on the Magellan $6.5 \mathrm{~m}$ Clay Telescope ; 2 - Alves-Brito et al. (2005) from UVES at VLT-ESO; 3 -Cordero et al. (2014) with data from Hydra multifiber spectrograph at Blanco $4 \mathrm{~m}$ telescope and FLAMES at VLT-ESO; 4 - Thygesen et al. (2014) from UVES at VLT-ESO; 5 - Carretta et al. (2009) mainly from UVES at VLT-ESO; 6 - Pancino et al. (2017) with data from Gaia-ESO survey, obtained with UVES and GIRAFFE at VLT; 7 - Horta et al. (2020) from near-infrared APOGEE data (Majewski, Schiavon \& Frinchaboy 2017); 8 - Wylie et al. (2006) from AGBs observed with the UCLES echelle spectrograph at the Anglo-Australian telescope; 9 and 10 Lapenna et al. (2014), from AGBs observed with FEROS at MPG/ESO; 11 - Colucci et al. (2017) from Du Pont telescope at Las Campanas; 12 - McWilliam \& Bernstein (2008) from Du Pont telescope at Las Campanas; 13 - Larsen et al. (2017) with data from UVES at VLT-ESO; 14 - Graves \& Schiavon (2008) with data from Schiavon et al. (2005), which used the R-C spectrograph at Blanco telescope in Cerro Tololo observatory; 15 - Conroy et al. (2018) with the same data from Schiavon et al. (2005); 16 - The compilation made by Roediger et al. (2014).

Table 2. Comparison with the abundances calculated in Colucci et al. (2017) for 47 Tuc. It is important to highlight that our adopted metallicity is $[\mathrm{Fe} / \mathrm{H}]=-0.78$, while in that work it was $[\mathrm{Fe} / \mathrm{H}]=-0.65$.

\begin{tabular}{lccccc}
\hline species & $\lambda(\AA)$ & $\mathrm{A}(\mathrm{X})_{\odot \text { Colucci }}$ & $\mathrm{A}(\mathrm{X})_{\text {Colucci }}$ & {$[\mathrm{X} / \mathrm{Fe}]_{\text {Colucci }}$} & {$[\mathrm{X} / \mathrm{Fe}]_{\text {thiswork }}$} \\
\hline $\mathrm{Na} \mathrm{I}$ & 5682.633 & 6.44 & 5.86 & +0.07 & +0.10 \\
$\mathrm{Na} \mathrm{I}$ & 5688.200 & 6.34 & 5.96 & +0.27 & +0.60 \\
$\mathrm{Na} \mathrm{I}$ & 6154.230 & 6.24 & 5.86 & +0.27 & +0.35 \\
$\mathrm{Mg}$ I & 5528.405 & 7.52 & 7.22 & +0.35 & +0.38 \\
$\mathrm{Al} \mathrm{I}$ & 6698.673 & 6.46 & 6.07 & +0.26 & +0.24 \\
Si I & 5948.55 & 7.61 & 7.23 & +0.27 & +0.42 \\
Si I & 7415.96 & 7.56 & 7.62 & +0.71 & +0.32 \\
Si I & 7423.51 & 7.60 & 7.43 & +0.48 & +0.12 \\
Ca I & 6102.723 & 6.45 & 5.83 & +0.03 & +0.24 \\
Ca I & 6166.440 & 6.36 & 5.83 & +0.12 & +0.24 \\
Ca I & 6439.080 & 6.02 & 5.63 & +0.26 & +0.02 \\
Ca I & 6455.605 & 6.29 & 5.73 & +0.09 & +0.15 \\
Ti I & 5866.449 & 5.04 & 4.57 & +0.18 & +0.10 \\
Ti I & 6556.077 & 5.41 & 4.77 & +0.01 & +0.32 \\
Ti II & 5336.077 & 5.15 & 4.77 & +0.27 & +0.37 \\
Ti II & 5381.021 & 5.21 & 4.67 & +0.11 & +0.37 \\
Eu II & 6645.064 & 0.42 & 0.14 & +0.37 & +0.32 \\
\hline
\end{tabular}

the WiFeS Atlas of Galactic Globular Cluster Spectra (WAGGS) survey.

The analysis is carried out as described in Moura et al. (2019). Basic parameters for 47 Tuc stem from literature ages from colourmagnitude diagrams (CMDs) and abundances both from individual stars and integrated spectra.
In Section 2, the method of building synthetic spectra is briefly described. In Section 3, the element abundances available in the literature are compiled. In Section 4, the different methods of analysis of integrated spectra applied to 47 Tuc are discussed. In Section 5, the results are presented and in Section 6, a summary is given. 

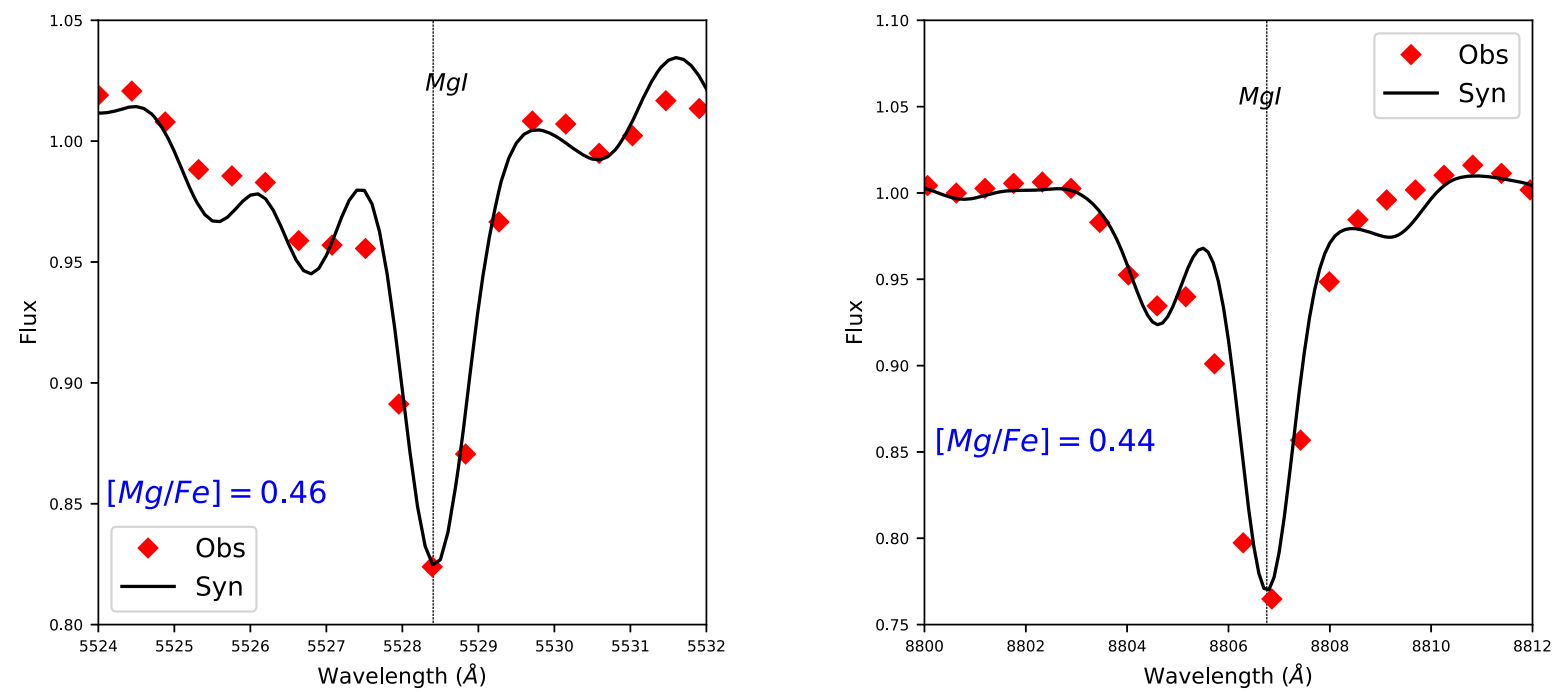

Figure 1. Observed and the best-fitting synthetic spectra for the $\mathrm{Mg}$ I lines at $5528.405 \AA$ (left-hand panel) and $8806.756 \AA$ (right-hand panel).
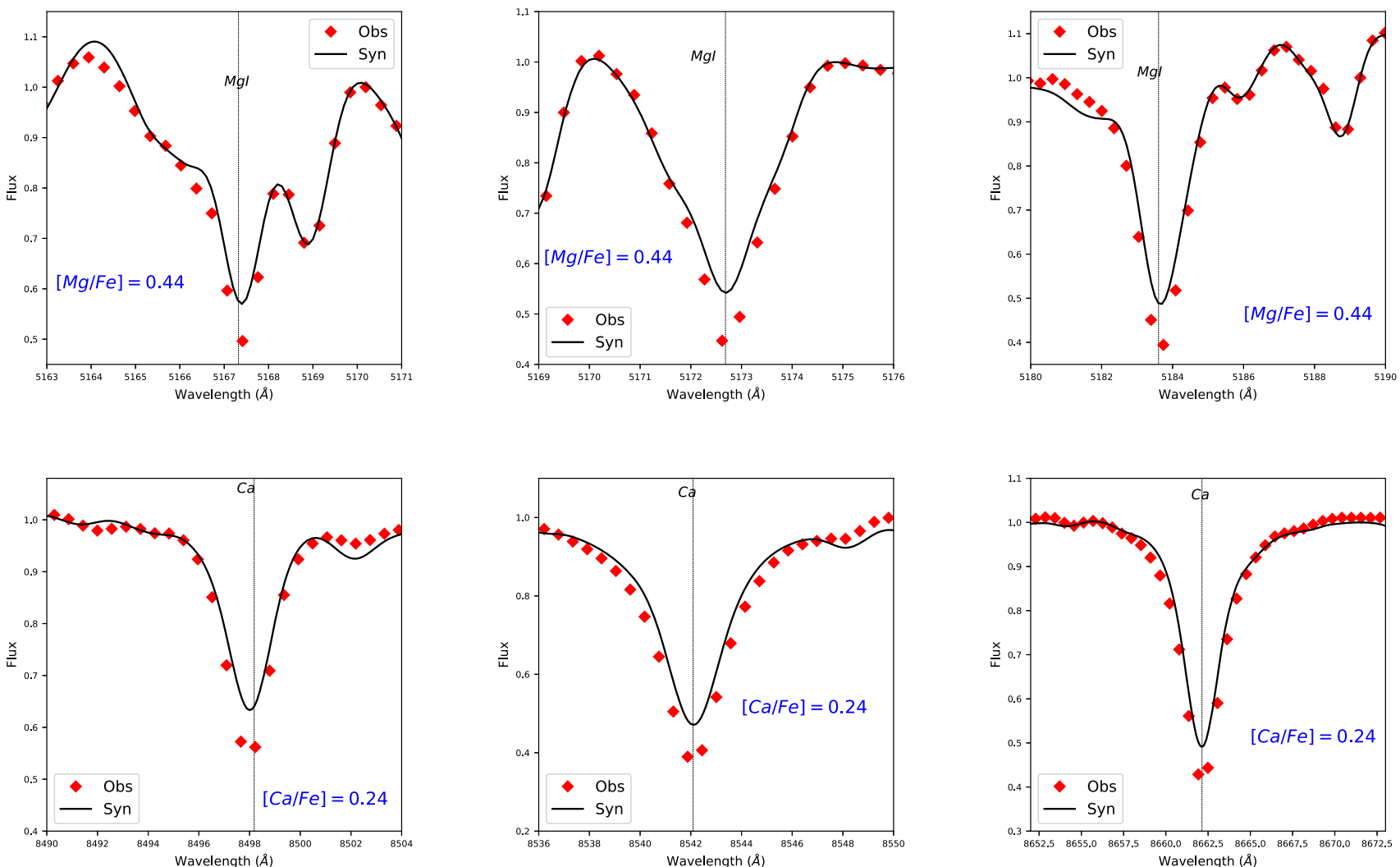

Figure 2. Observed and best-fitting synthetic spectra for the Mg I and Ca II (lower panels) strong lines. The Mg I triplet lines at 5167.32, 5172.68, and 5183 are shown in the upper panel, and in the bottom panels, we show the CaI triplet lines at 8498.18, 8542.089, and 8662.14 $\mathrm{A}$.

\section{BUILDING SYNTHETIC INTEGRATED SPECTRA}

In this work, we use the code SYNSSP, that combines the PFANT code for spectrum synthesis of individual stars (Barbuy, Trevisan \& de Almeida 2018a), with a stellar evolutionary model for a given age, metallicity, and helium abundance. A first version of SYNSSP was employed in La Barbera et al. (2013), where the aim was to test the effect of individual variations of $\mathrm{Ca}, \mathrm{Na}$, and $\mathrm{Ti}$ abundances on the indices studied $\mathrm{CaH} \& \mathrm{~K}, \mathrm{NaD}, \mathrm{Na} 8190, \mathrm{TiO}_{1}, \mathrm{TiO}_{2}$. An updated version, described in detail, is given in Moura et al. (2019). A brief description of SYNSSP can be summarized as follows: SYNSSP builds the spectrum of a Single Stellar Population (SSP) with the following input data: (a) $[\mathrm{Fe} / \mathrm{H}],[\alpha / \mathrm{Fe}]$, and age of the SSP; (b) number of stars considered along the isochrone, from the main sequence (MS), subgiant branch (SGB), and RGB. The standard procedure considers $12 \mathrm{MS}$ and SGB stars and 9 RGB stars; (c) solar abundances of all elements and abundance ratios relative to solar; (d) wavelength interval to be generated and spectral resolution 

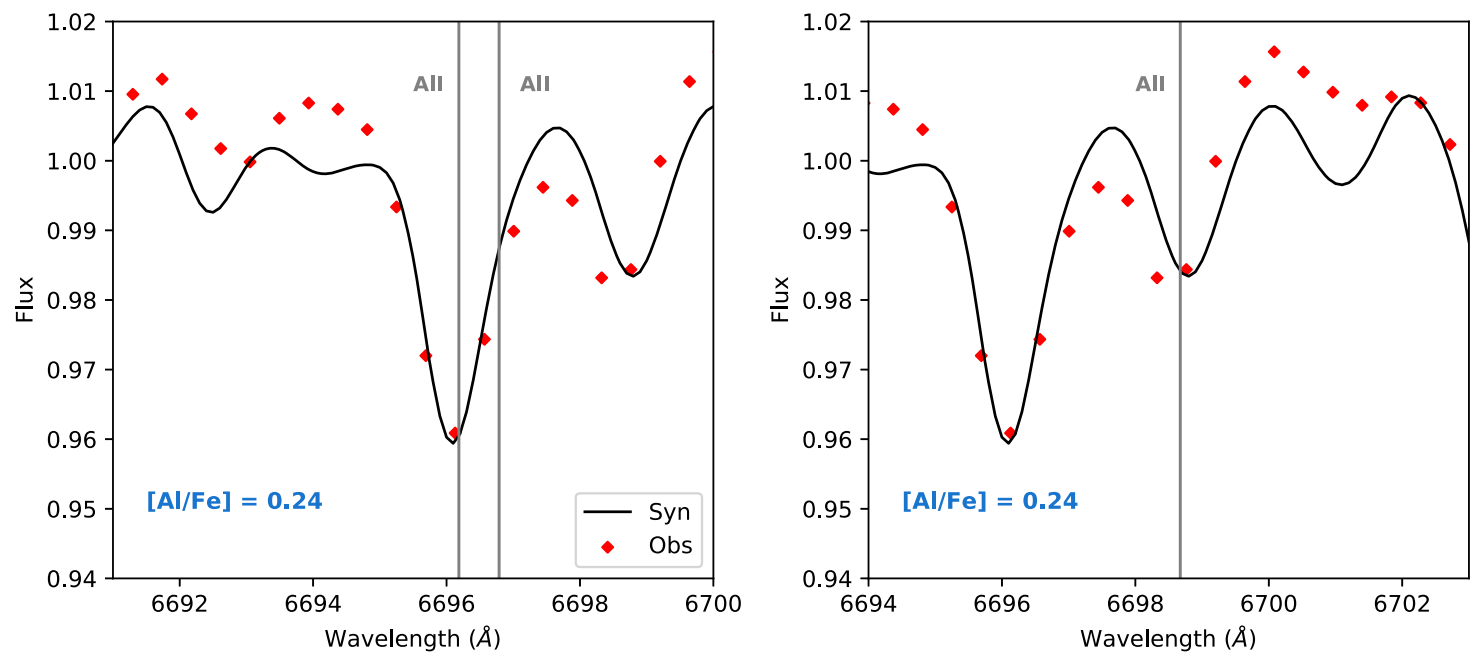

Figure 3. Observed and best-fitting synthetic spectra for the Al I lines at 6696.185 and $6696.788 \AA$ (left-hand panel) and $6698.673 \AA$ (right-hand panel).
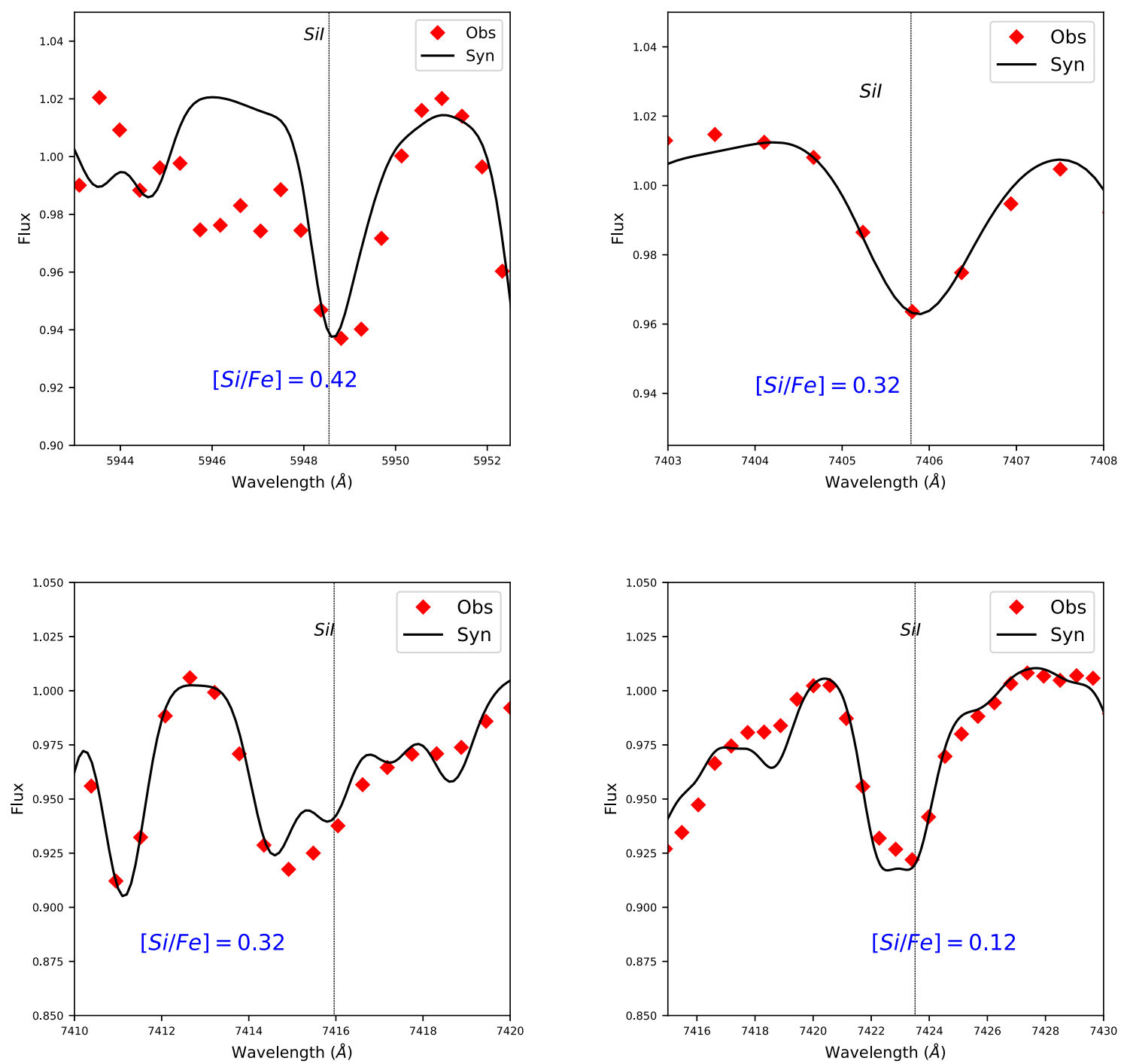

Figure 4. Observed and best-fitting synthetic spectra for the Si I lines at $5948.55 \AA$ (upper left-hand panel), $7405.79 \AA$ (upper right-hand panel), $7415.96 \AA$ (lower left-hand panel), and $7423.51 \AA$ (lower right-hand panel). 

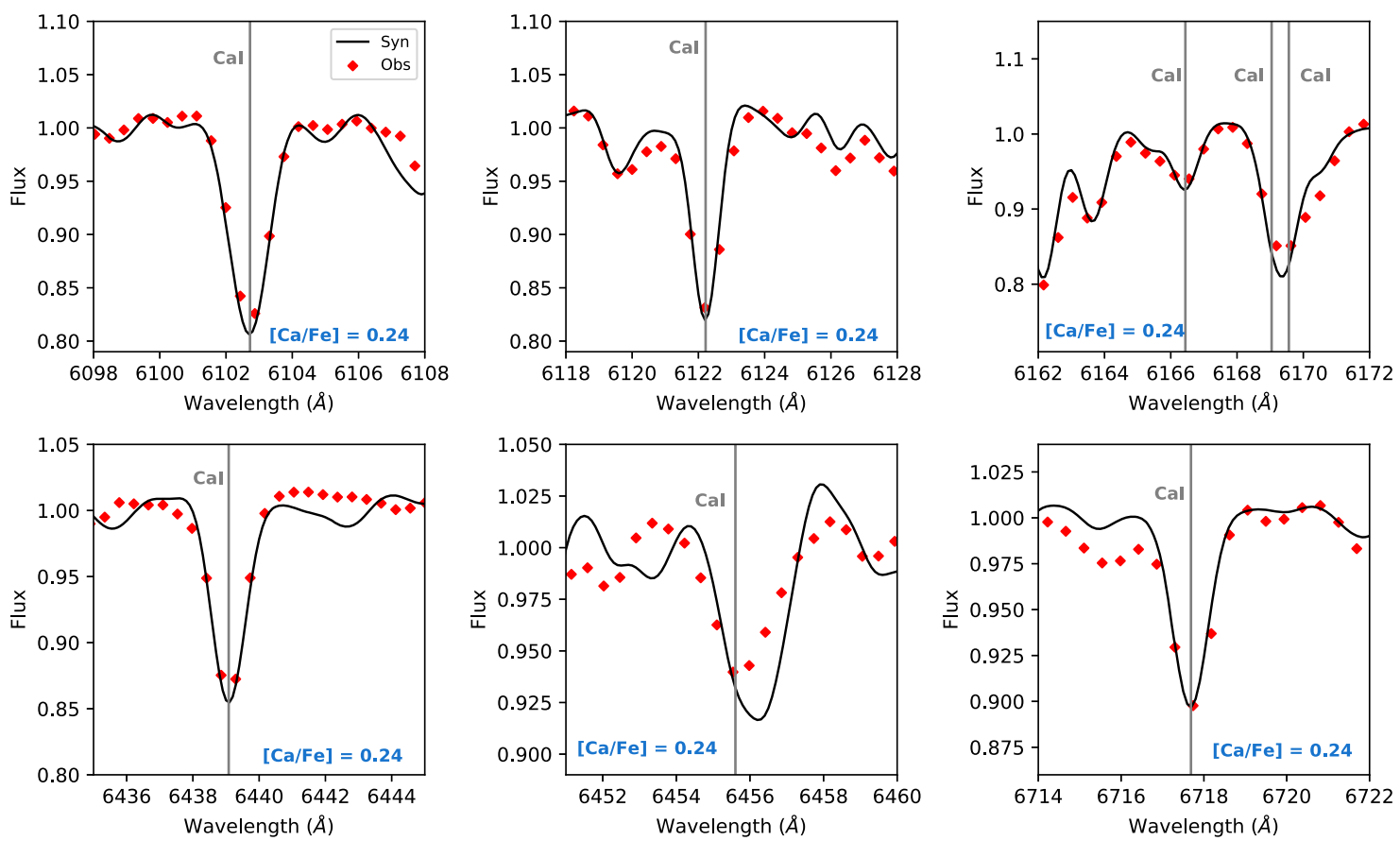

Figure 5. Observed and best-fitting synthetic spectra for the Ca I lines.

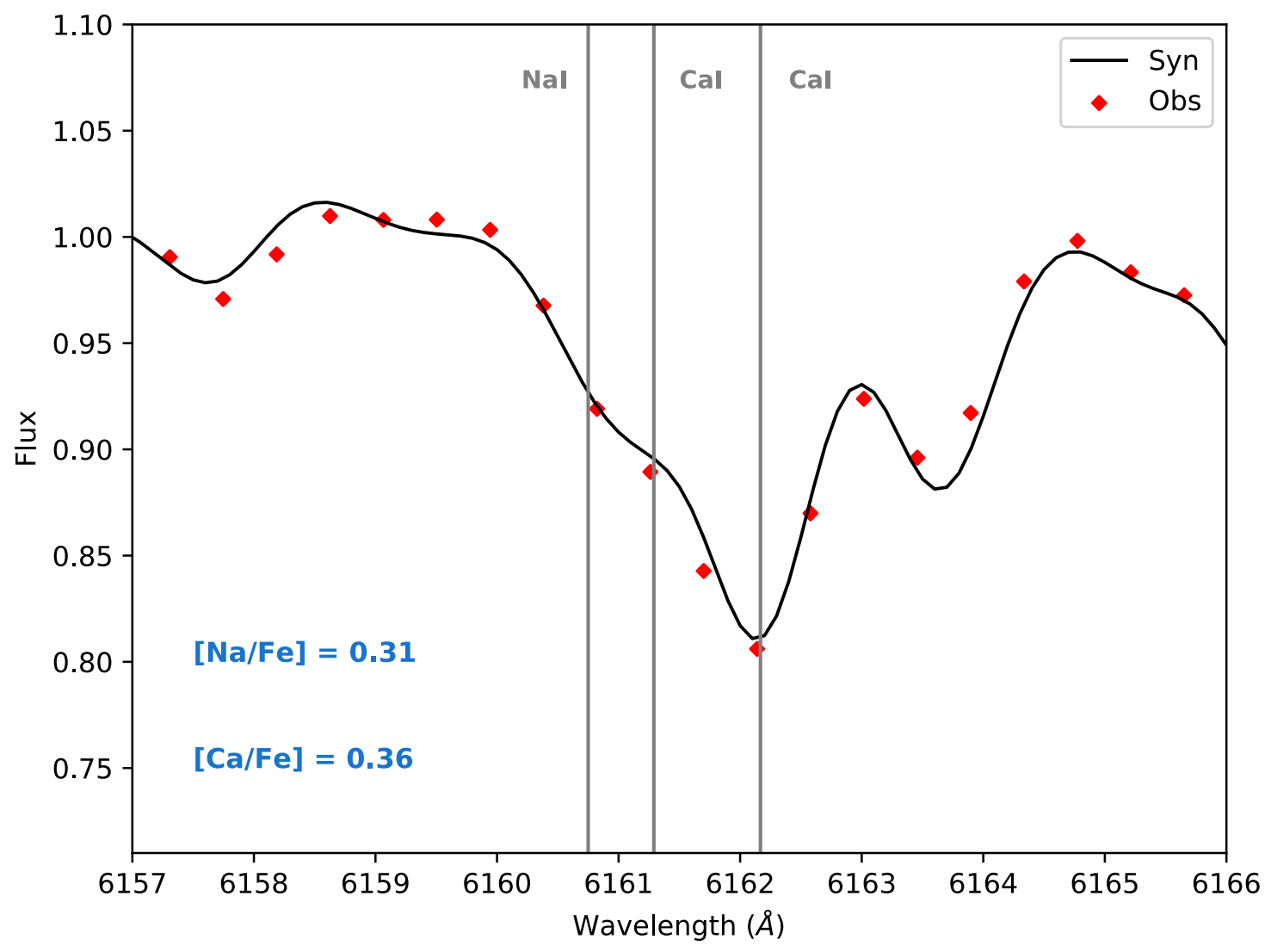

Figure 6. Observed and synthetic spectra fitting for the Ca I lines at 6161.295 and $6162.167 \AA$ and the Na I line at $6160.753 \AA$.

(or FWHM) desired. The standard value is FWHM $=1.0 \AA$ that fits well the Usher et al. (2017) spectra, having a resolution of $\mathrm{R}$ $\sim 6800$.
We use Dartmouth isochrones (Dotter et al. 2008), with $[\alpha / \mathrm{Fe}]$ $=+0.4$ and $[\mathrm{Fe} / \mathrm{H}]=-0.8$. From the evolutionary tracks, $21 \log g$ $-T_{\text {eff }}$ pairs along the isochrone are identified, from the tip of the 

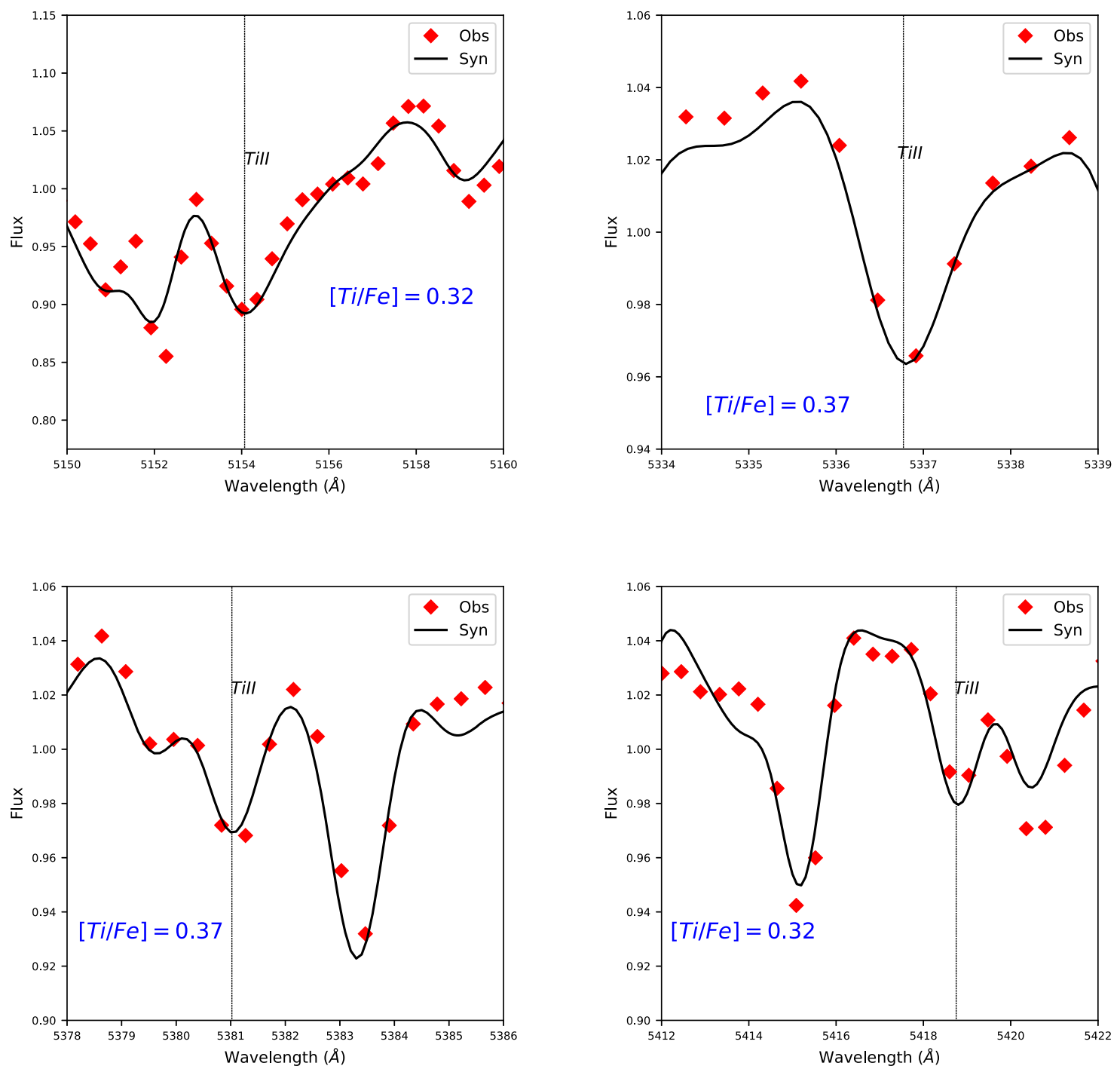

Figure 7. Observed and best-fitting synthetic spectra for the Ti II lines at $5154.068 \AA$ (upper left-hand panel), $5336.771 \AA$ (upper right-hand panel), $5381.021 \AA$ (lower left-hand panel) and $5418.751 \AA$ (lower right-hand panel).

RGB $\left(T_{\text {eff }}=3000 \mathrm{~K}\right.$ and $\left.\log g=-0.1\right)$ down to the main-sequence locus $\left(T_{\text {eff }}=3400 \mathrm{~K}\right.$ and $\left.\log g=5.2\right)$. For each $\log g-T_{\text {eff }}$ pair, we generate synthetic stellar spectra with the PFANT code described in Barbuy et al. (2018a), and references therein. Given a stellar model atmosphere and lists of atomic and molecular lines, the code computes a synthetic spectrum assuming local thermodynamic equilibrium (LTE). We use the MARCS 1D LTE atmospheric models (Gustafsson et al. 2008). For $\log g>3$, the atmospheric model geometry is plane-parallel and mass independent; for $\log g<$ 3 (giants with non-negligible photospheric depths), the models are calculated for a spherical geometry of $1 \mathrm{M}_{\odot}$ stars. A microturbulent velocity of $2 \mathrm{~km} \mathrm{~s}^{-1}$ is adopted for all spectra. Finally, the SSP spectra are created by combining all the synthetic spectra, which are divided in selected bins, each of them with its specfic weight, following the expression

$F_{\mathrm{SSP}}(\lambda)=\frac{\sum_{i=1}^{n_{\text {bins }}} S_{\star, i}(\lambda) f_{\text {corr }, i} N_{i}}{\sum_{i=1}^{n_{\text {bins }}} M_{i}}$

where $n_{\text {bins }}$ is the number of bins along the isochrone and $S_{\star, i}(\lambda)$ is the spectrum of the star in the centre of the bin, in units of $\mathrm{L}_{\odot} / \AA$. The number of stars, $N_{i}$, and the total stellar mass, $M_{i}$ (given in units of $\mathrm{M}_{\odot}$ ), of each bin are obtained by integrating the initial mass function (IMF) from $M_{l, i}$ to $M_{u, i}$ (the mass limits of the bin)

$$
\begin{aligned}
N_{i} & =\int_{M_{l, i}}^{M_{u, i}} \Phi(m) \mathrm{d} m, \\
M_{i} & =\int_{M_{l, i}}^{M_{u, i}} m \Phi(m) \mathrm{d} m .
\end{aligned}
$$

Finally, the correction factor $f_{\text {corr, } i}$ accounts for the variation of stellar luminosities within the bin and is given by $f_{\text {corr, } i}=L_{\text {total, }, \mathrm{i}} / L_{i}$, where

$$
\begin{aligned}
L_{\mathrm{total}, i} & =\int_{M_{l, i}}^{M_{u, i}} L(m) \Phi(m) \mathrm{d} m, \\
L_{i} & =L_{\star, i} \int_{M_{l, i}}^{M_{u, i}} \Phi(m) \mathrm{d} m,
\end{aligned}
$$

i.e. the ratio between the luminosity of the bin and the luminosity of the bin assuming that $L(m)=$ constant $=L_{\star, i}$ (the luminosity of the star for which we computed the spectrum $S_{\star, i}$ ). The units of the final SSP spectrum in equation (1) are $\mathrm{L}_{\odot} /\left(\mathrm{M}_{\odot} \AA\right)$. We adopt 

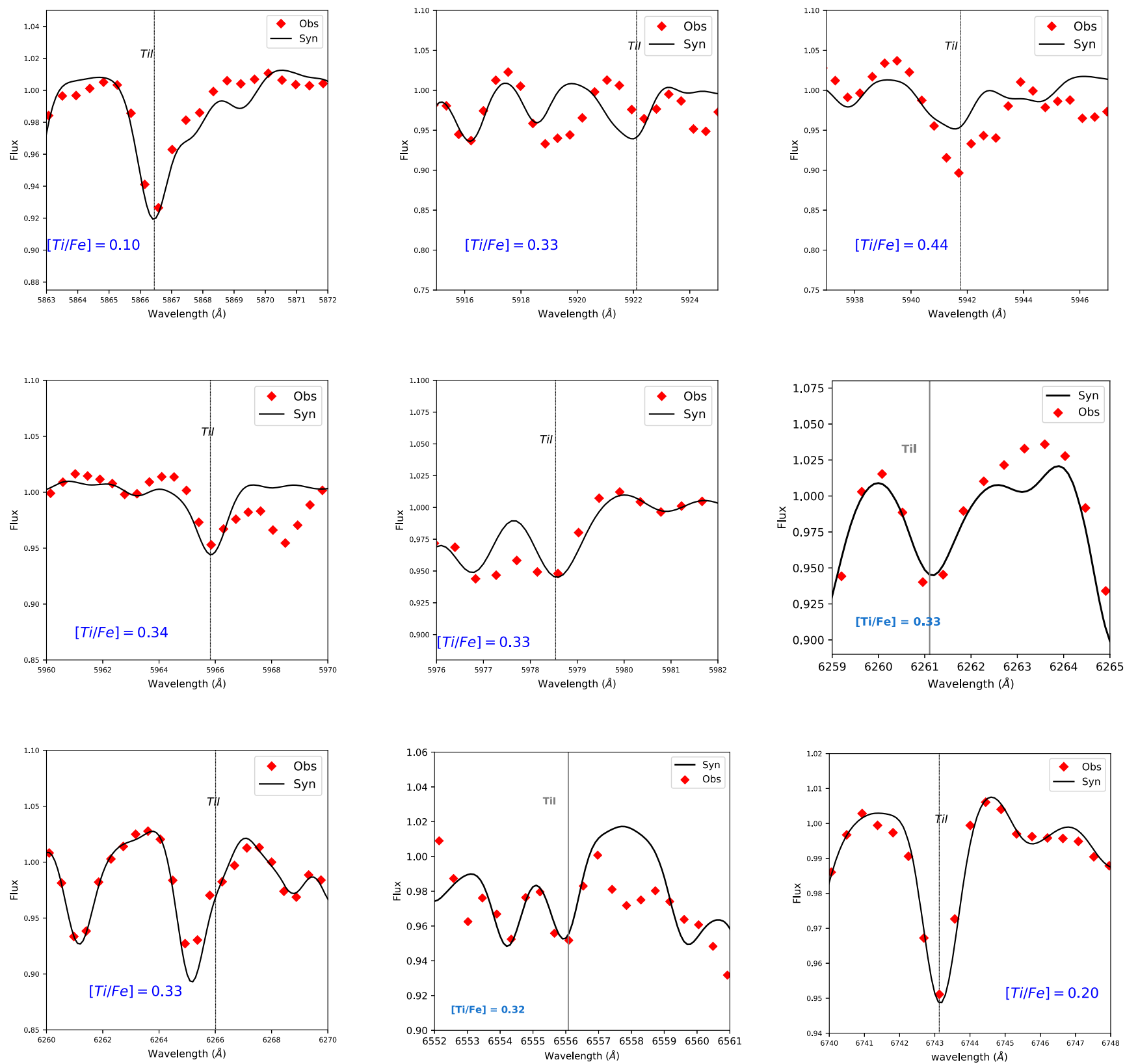

Figure 8. Observed and best-fitting synthetic spectra for the Ti I lines.

a mass interval from $M_{1}=0.15 \mathrm{M}_{\odot}$ to $M_{2}=1.01 \mathrm{M}_{\odot}$, and a Salpeter IMF (Salpeter 1955). Finally, the input observed spectrum is normalized through a polynomial fitting to match the continuum of the computed spectrum.

\section{LITERATURE ABUNDANCES IN 47 TUCANAE}

Among the first studies regarding chemical abundances in globular clusters were the ones made by Pilachowski et al. (1980), that identified chemical enhancement in the $\mathrm{CN}$ and $\mathrm{CO}$ bands in two clusters including 47 Tuc, using HRS and photometry; Dickens et al. (1979) found an overabundance of $\mathrm{N}$ in red giants observed in 47 Tuc based on low-resolution spectra; Norris \& Cottrell (1979) also studied the nitrogen anomaly in 47 Tuc by observing two red giants with spectra at a resolution around $1 \AA$ A. More recently, after the development of the 8-10 m telescopes, HRS made possible more detailed and precise determinations of chemical abundances of individual stars in globular clusters, that can be used as tracers of the chemical abundances of the clusters themselves. Different types of stars have been observed, such as RGB stars (Koch \& McWilliam 2008; Alves-Brito et al. 2005; Thygesen et al. 2014; Cordero et al. 2014; Carretta et al. 2009), subgiants and dwarfs (Carretta et al. 2004), and AGBs (Wylie et al. 2006; Lapenna et al. 2014). From analyses of high-resolution spectra of RGBs, metallicities in the range $-0.66<[\mathrm{Fe} / \mathrm{H}]<-0.79$ are found, giving a mean value of $[\mathrm{Fe} / \mathrm{H}] \sim-0.75$.

In Table 1, the literature abundances in 47 Tucanae are reported, together with the method employed. The studies comprise HRS of individual RGB and AGB stars and analysis of integrated spectra with the methods of: measurement of Lick indices (ISLick), measurement of EWs (ISEW), full spectrum fitting (ISFF), or fit of 

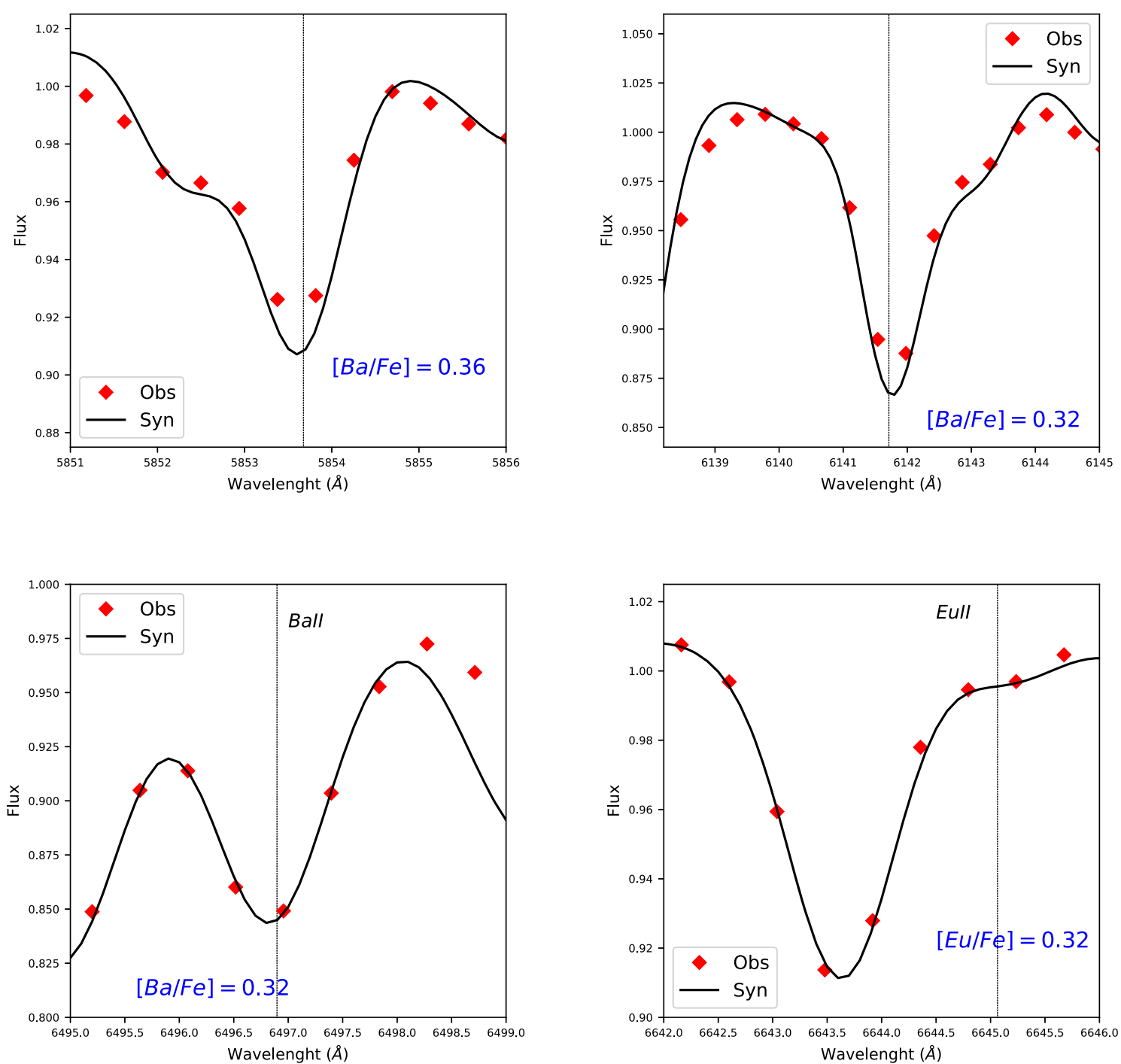

Figure 9. Observed and synthetic spectra for the 5853.675, 6141.713, and $6496.9 \AA$ for Ba II lines and Eu II $6645.064 \AA$ line.

individual lines (ISfit) (the latter being our adopted method), or compilations.

\section{PREVIOUS METHODS APPLIED TO 47 TUC}

Different methods and different wavelength ranges have been applied to interpret integrated spectra of 47 Tuc.

Graves \& Schiavon (2008) employed the EZ_AGES code based on Schiavon (2007) models, combined with Padova isochrones, in two modes, one with solar-scaled abundances (Girardi et al. 2000) and the other with $\alpha$-enhancement $[\alpha / \mathrm{Fe}]=0.42$ (Salasnich et al. 2000). Input abundances of $[\mathrm{Mg} / \mathrm{Fe}],[\mathrm{C} / \mathrm{Fe}],[\mathrm{N} / \mathrm{Fe}],[\mathrm{O} / \mathrm{Fe}],[\mathrm{Ca} / \mathrm{Fe}],[\mathrm{Na} / \mathrm{Fe}]$, $[\mathrm{Si} / \mathrm{Fe}],[\mathrm{Cr} / \mathrm{Fe}]$, and $[\mathrm{Ti} / \mathrm{Fe}]$ are included independently, in order to model Lick indices measurements for different ages and values of $[\mathrm{Fe} / \mathrm{H}]$ and compare these with measured integrated spectra. The lines employed to derive ages and $[\mathrm{Fe} / \mathrm{H}]$ are the ones that are strongly affected by the variations of these two parameters, whereas insensitive to variations on element abundances. The code includes ages in the range of $0.1 \leq t \leq 15.8 \mathrm{Gyr}$, metallicities within $-1.3 \leq[\mathrm{Fe} / \mathrm{H}]$ $\leq+0.2$ for the solar-scaled isochrone and $-0.8 \leq[\mathrm{Fe} / \mathrm{H}] \leq+0.3$ for the $\alpha$-enhanced ones. The Lick indices studied were $\mathrm{H} \delta_{\mathrm{A}}$ (wide definitions for A stars), $\mathrm{H} \delta_{\mathrm{F}}$ (narrow definition for $\mathrm{F}$ stars and later),
$\mathrm{H} \gamma_{\mathrm{A}}, \mathrm{H} \gamma_{\mathrm{F}}, \mathrm{H} \beta, \mathrm{CN}_{1}, \mathrm{CN}_{2}, \mathrm{Ca} 4227, \mathrm{G} 4300, \mathrm{Fe} 4383, \mathrm{C} 24668$, $\mathrm{Fe} 5015, \mathrm{Mg}_{2}, \mathrm{Mg} b, \mathrm{Fe} 5270$, and $\mathrm{Fe} 5335$. These indices are as defined in Worthey et al. (1994) and Worthey \& Ottaviani (1997). The indices calculated can be compared with measured indices from observed spectra to then determine the best-fitting mean age and $[\mathrm{Fe} / \mathrm{H}]$ for the stellar population, for a given set of model abundance ratios.

McWilliam \& Bernstein (2008) employed a different approach, adopting empirical CMDs and EWs in the construction of the synthetic spectra. Their technique was based in dividing the CMD in multiple regions with different types of stars and calculating synthetic EWs for each line, for each CMD bin. The final EWs are obtained from a weighted combination of the bin fluxes. The abundances were calculated by iteratively fitting synthetic flux-weighted EWs to the observed spectra, until it matches the observed integrated light (IL) spectra. For measuring the EWs in the IL spectra, the GETJOB code (McWilliam et al. 1995) was used. Finally, in order to obtain the best fits, their new package ILABUNDS was employed, which includes $\alpha$ enhanced model atmosphere grids from Kurucz (Castelli \& Kurucz 2004) and the MOOG code (Sneden 1973) for spectrum synthesis. Their linelist includes lines of the elements $\mathrm{Na}, \mathrm{Mg}, \mathrm{Al}, \mathrm{Si}, \mathrm{Ca}, \mathrm{Sc}$, $\mathrm{Ti}, \mathrm{V}, \mathrm{Cr}, \mathrm{Mn}, \mathrm{Fe}, \mathrm{Co}, \mathrm{Ni}, \mathrm{Cu}, \mathrm{Y}, \mathrm{Zr}, \mathrm{Ba}, \mathrm{La}, \mathrm{Nd}$, and $\mathrm{Eu}$ in the wavelength range 5000-7570 ̊. 


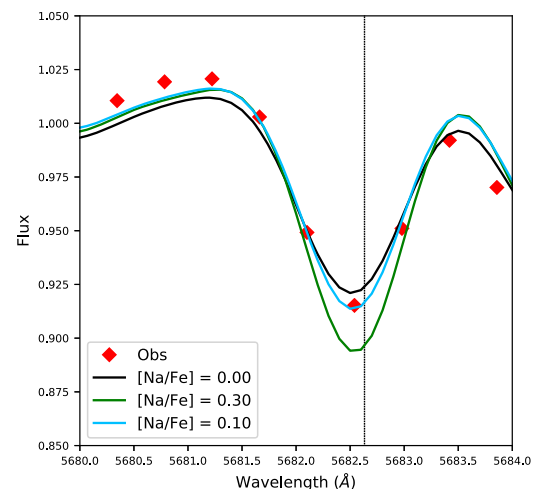

Wavelength $(\AA)$

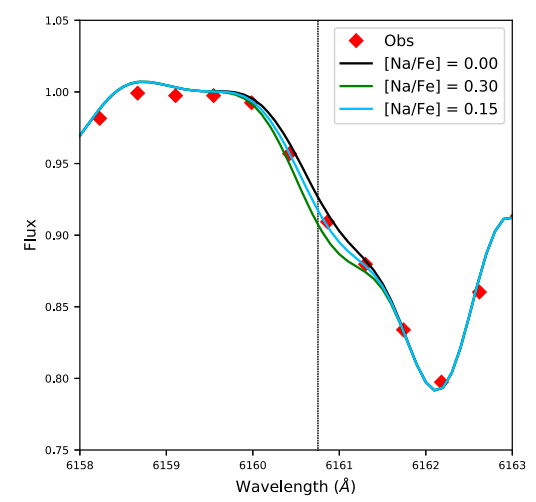

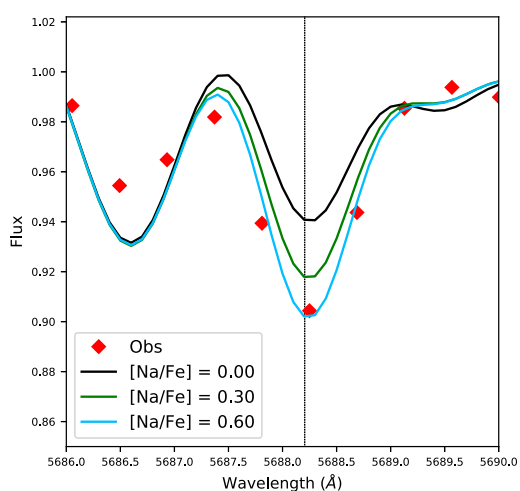
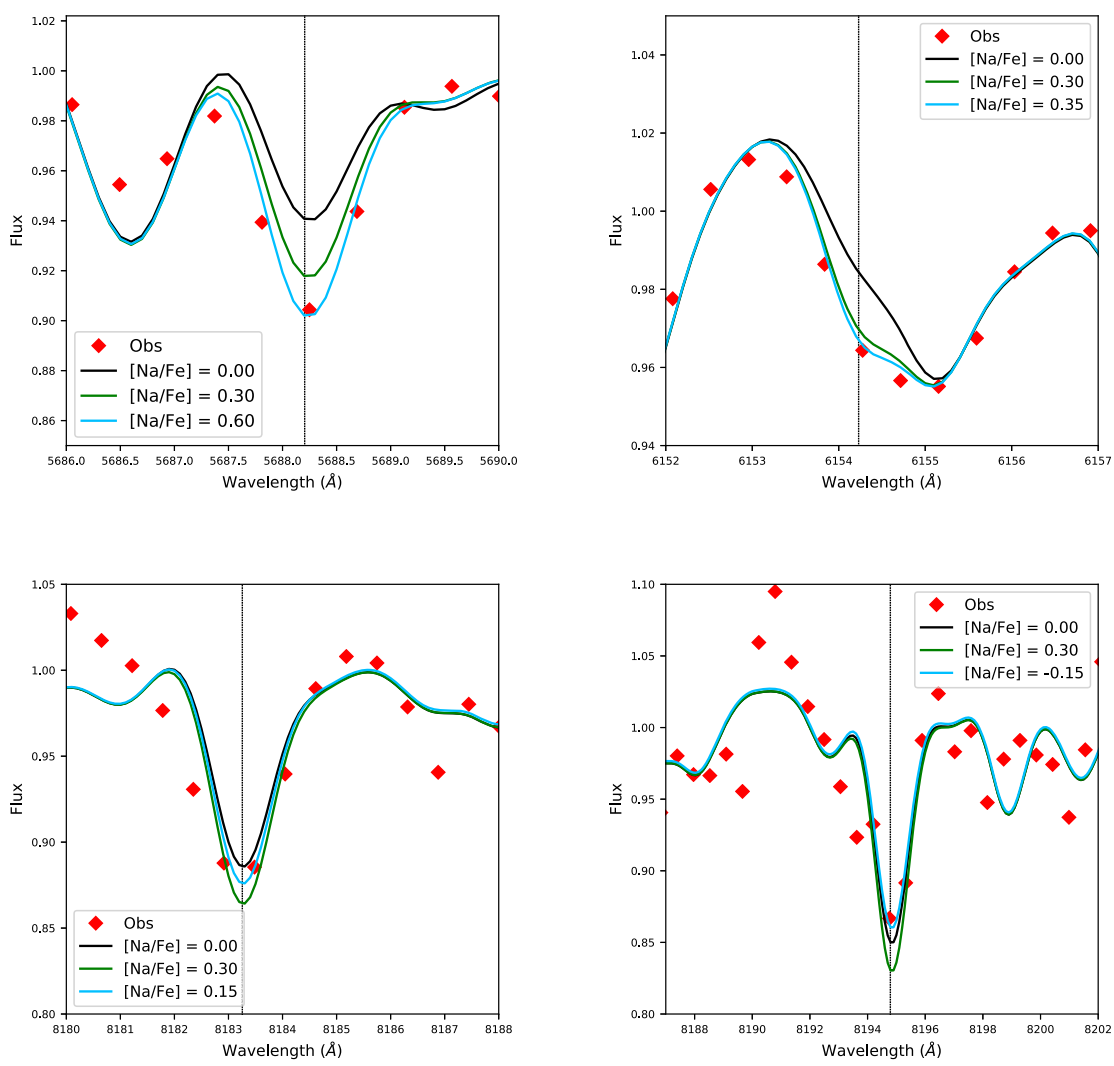

Figure 10. Observed and $[\mathrm{Na} / \mathrm{Fe}]=0.00,0.30$ and best-fitting synthetic spectra for the $\mathrm{Na}$ I lines.

Colucci et al. (2017) analysis of 47 Tuc employed the same techniques previously used in McWilliam \& Bernstein (2008), with a change of stellar evolution models. For the calculation of integrated EWs, in the different CMD bins, the BaSTI isochrones from the Teramo group (Pietrinferni et al. 2004, 2006; Cordier et al. 2007), and a Kroupa IMF (Kroupa 2002, IMF) were adopted. Some lines used by them are also studied here, therefore a comparison between their abundances and the present ones is given in Table 2.

Larsen et al. (2017) employs full spectrum fitting to high resolution integrated spectra $(\mathrm{R} \sim 40000)$ for derivation of elemental abundances in 47 Tuc. The abundances were extracted from the best fitting of multiple lines simultaneously, i.e. the fitting of the synthetic to the observed spectra is carried out by iteratively fitting the abundances until the best overall fit for all lines is reached. Both theoretical isochrones and empirical CMDs were adopted, in both cases divided in bins of stars. The observed CMDs are photometric data from ACS/HST (Sarajedini et al. 2007; Anderson et al. 2008). The theoretical Dartmouth $\alpha$-enhanced isochrones were employed only for the least luminous bins. In their analysis, they found that using stars based on theoretical isochrones give very similar results to those based on resolved CMDs. A Salpeter IMF and MARCS model atmospheres with the TURBOSPECTRUM code (Alvarez \& Plez 1998; Plez 2012) were adopted.

Conroy et al. (2018) also apply full spectral fitting techniques for measurement of abundances in 47 Tuc and 40 other clusters. Their approach includes the Dartmouth isochrones, Kroupa IMF, and the libraries of spectra MILES (Sánchez-Blázquez et al. 2006) and Extended IRTF empirical spectra (Prugniel, Vauglin \& Koleva 2011; Sharma, Prugniel \& Singh 2016), complemented with a library of M dwarfs (Mann et al. 2015). A polynomial spectral interpolator assigns each point from the isochrone to a suitable stellar spectrum from the libraries. Because such libraries are restricted in variations of abundances due to relying on empirical star spectra, response functions were developed to address this problem. Their SSPs are compared with the empirical spectra from Schiavon et al. (2005) and abundances of $\mathrm{Mg}, \mathrm{Si}, \mathrm{Ca}$, and $\mathrm{Ti}$ were computed.

\section{RESULTS AND DISCUSSION}

After carrying out calculations for the overall spectrum with the metallicities and abundances from the first seven references given in Table 1, we chose the results by Thygesen et al. (2014) as best fitting and therefore adopted a metallicity $[\mathrm{Fe} / \mathrm{H}]=-0.78$ and $[\alpha / \mathrm{Fe}]=$ +0.4 as fixed input data. These values are close to most of the other derivations from optical high-resolution data, with $[\mathrm{Fe} / \mathrm{H}]=-0.76$ from Koch \& McWilliam (2008), $[\mathrm{Fe} / \mathrm{H}]=-0.79$ from Cordero et al. (2014), $[\mathrm{Fe} / \mathrm{H}]=-0.77$ from Carretta et al. (2009) - see Table 1.

As for the $[\alpha / \mathrm{Fe}]$ values, it is interesting to note that, according to Milone et al. (2017) in 47 Tuc, only about $\sim 20$ per cent of stars belong to a first stellar generation $(1 \mathrm{G})$, whereas the $\sim 80$ per cent of stars from a second generation $(2 \mathrm{G})$ might show low oxygen abundances. However, it is not clear if most of $2 \mathrm{G}$ stars would have low oxygen abundances and all derivations in Table 1 indicate high oxygen abundances, therefore we find reasonable to keep a high $[\alpha / \mathrm{Fe}]$ value. A confirmation that this is the correct choice, is the mean abundance of the $\mathrm{Mg}$ of $[\mathrm{Mg} / \mathrm{Fe}]=0.45$, derived in this work from the integrated spectrum. We recall that $\mathrm{O}$ and $\mathrm{Mg}$ are the bona fide alpha-elements produced during hydrostatic burning in the interior of stars, whereas $\mathrm{Si}, \mathrm{Ca}$, and $\mathrm{Ti}$ are mostly produced during explosive burning in supernovae events (e.g. McWilliam 2016; Barbuy et al. 2018b).

For the isochrone, an age of $11 \mathrm{Gyr}$ was adopted, as a mean value from ages assigned to 47 Tuc reported in Table 3 . There is 
Table 3. Literature ages (Gyr) for 47 Tuc.

\begin{tabular}{lcc}
\hline Age $(\mathrm{Gyr})$ & Method & Reference \\
\hline 11 to $\sim 13$ & Spectroscopy & Schiavon et al. (2002) \\
11.3 & Main-sequence fitting & Gratton, Bragaglia \& Carretta (2003) \\
11.5 & Main-sequence fitting & Dotter et al. (2009) \\
11.25 & Eclipsing binary star (V69) & Thompson et al. (2010) \\
9.7 & White dwarf cooling sequence & Hansen et al. (2013) \\
11.75 & Main-sequence fitting & VandenBerg et al. (2013) \\
12 & Main-sequence fitting & VandenBerg et al. (2014) \\
13 & Main-sequence fitting & Denissenkov et al. (2017) \\
11.8 & MSF and V69 analysis & Brogaard et al. (2017) \\
\hline
\end{tabular}

Table 5. Abundances derived for $\mathrm{Mg}, \mathrm{Al}, \mathrm{Si}, \mathrm{Ca}, \mathrm{Ti}, \mathrm{Ba}$ and $\mathrm{Eu}$.

\begin{tabular}{lccccccc}
\hline$[\mathrm{Mg} / \mathrm{Fe}]$ & {$[\mathrm{Al} / \mathrm{Fe}]$} & {$[\mathrm{Si} / \mathrm{Fe}]$} & {$[\mathrm{Ca} / \mathrm{Fe}]$} & {$[\mathrm{Ti} / \mathrm{Fe}](\mathrm{Ti})$} & {$[\mathrm{Ti} / \mathrm{Fe}](\mathrm{Ti} \mathrm{II})$} & {$[\mathrm{Ba} / \mathrm{Fe}]$} & {$[\mathrm{Eu} / \mathrm{Fe}]$} \\
\hline $0.45 \pm 0.01$ & $0.24 \pm 0.01$ & $0.30 \pm 0.07$ & $0.24 \pm 0.07$ & $0.30 \pm 0.03$ & $0.35 \pm 0.02$ & $0.33 \pm 0.02$ & 0.32 \\
\hline
\end{tabular}

little change in the resulting integrated synthetic spectra, from 11 to $13 \mathrm{Gyr}$, given that the RGB stars dominate the flux and changes in the turn-off point with age have little effect on the overall flux. This is shown in Moura et al. (2019), where fig. 14 shows the comparison of spectra between 11 and 13 Gyr for NGC 6522 .

The abundances were derived similarly to methods employed with high-resolution spectra, i.e. by fitting the continuum of the synthetic to the observed spectrum locally and varying the abundance to find the best match to the line in question. The fits to the lines and corresponding adopted abundances in each case are shown in Figs 1-10 and comments on the results for the lines of each element are given below.

Table 5 lists the species, wavelength $(\AA)$, excitation potential $\chi_{\text {ex }}$ $(\mathrm{eV})$, oscillator strength log gf, van der Waals interaction constant $\mathrm{C}_{6}$, and abundances line-by-line. The damping constants are derived following Anstee \& O'Mara (1995), Barklem \& O'Mara (1997) and Barklem, O'Mara \& Ross (1998). The interaction constant $C_{6}$ relates to the damping constant through the formula $\gamma_{6} / N_{\mathrm{H}}=17 v^{3 / 5} C_{6}^{2 / 5}$, where $v$ is the velocity and $N_{\mathrm{H}}$ is the density of hydrogen atoms. For the strong triplet lines of $\mathrm{Mg}$ I and $\mathrm{Ca}$ I, the $\mathrm{C}_{6}$ values correspond to fits to wings of these lines in the solar and Arcturus spectra - see Moura et al. (2019).

The fit to the overall WAGGS spectrum of 47 Tuc in the wavelength range considered here is shown in Figs A1-A8 in the Appendix.

\section{$5.1 \mathrm{Mg}$ Lines}

Mg I 5528.405 and $8806.756 \AA$ lines fitted in Fig. 1 indicate $[\mathrm{Mg} / \mathrm{Fe}]=+0.45$. The $\mathrm{Mg}$ I triplet lines at $5167-5183 \AA$ are well fitted with this abundance, as can be seen in Fig. 2, where the oscillator strengths are from Pehlivan Rhodin et al. (2017) and the wings were fitted as described in Moura et al. (2019). The bottom of strong lines such as the Mg I triplet lines cannot be computed in LTE, because they form in NLTE regimen, and in chromospheric layers, that are not included in photospheric models.

\subsection{Al Lines}

We fitted the AlI 6696.185, 6696.788, 6698.673 $\AA$ lines, as shown in Fig. 3, and the best fit is obtained with $[\mathrm{Al} / \mathrm{Fe}]=+0.24$.

\subsection{Si Lines}

The best-fitting synthetic spectra for Si I lines are shown in Fig. 4. The four lines indicate a mean value of $[\mathrm{Si} / \mathrm{Fe}]=+0.30 \pm 0.07$.

\subsection{Ca Lines}

The Ca I lines at 6102.723, 6122.217, 6166.44, 6169.044, 6439.08, 6455.605, and 6717.687 $\AA$ are shown in Fig. 5. The lines at 6161.295 and $6162.167 \AA$ are shown in Fig. 6. A mean value of $[\mathrm{Ca} / \mathrm{Fe}]=$ $+0.24 \pm 0.07$ is obtained. This result is in very good agreement with the derived $\mathrm{Ca}$ abundance of $[\mathrm{Ca} / \mathrm{Fe}]=+0.20$ by Usher et al. (2019).

The CaT triplet lines are computed with $[\mathrm{Ca} / \mathrm{Fe}]=+0.24$, as shown in Fig. 2. Again, similarly to the case of the $\mathrm{Mg}$ I triplet, the bottom of these strong lines cannot be reproduced in LTE.

\subsection{Ti Lines}

The measurement of the titanium abundances was done in two separate groups of lines, being one based on the Ti II lines and the other on Ti I lines. The plots are shown in Figs 7 and 8, respectively. The Ti II lines give $[\mathrm{Ti} / \mathrm{Fe}]=+0.35 \pm 0.02$ and the neutral species give $[\mathrm{Ti} / \mathrm{Fe}]=0.30 \pm 0.03$, showing a good agreement among the different ionization stages. This is important because it is essentially a measure of the suitability of the effective temperatures adopted.

\subsection{Ba and Eu Lines}

The best-fits to the Ba II $6496.9 \AA$ and Eu II $6645.064 \AA$ lines are shown in Fig. 9. Hyperfine structure is taken into account as described in Moura et al. (2019).

\subsection{Multiple stellar populations}

The early work on $\mathrm{CN}$-weak and $\mathrm{CN}$-strong stars in globular clusters (e.g. Osborn 1971) and also see Section 3, was not interpreted as due to Multiple Stellar Populations (MPs), which took place in the years 2000s only. MPs have been clearly identified from photometry by Lee, Joo \& Sohn (1999) and Bedin et al. (2004), and from spectroscopy through $\mathrm{Na}-\mathrm{O}, \mathrm{Mg}-\mathrm{Al}$ anticorrelations, together with enhancements of $\mathrm{N}$ and deficiency in $\mathrm{O}$, that were systematically found in globular clusters (see reviews by Gratton et al. 2004; Gratton, Carretta \& Bragaglia 2012). Piotto et al. (2015) and Milone 


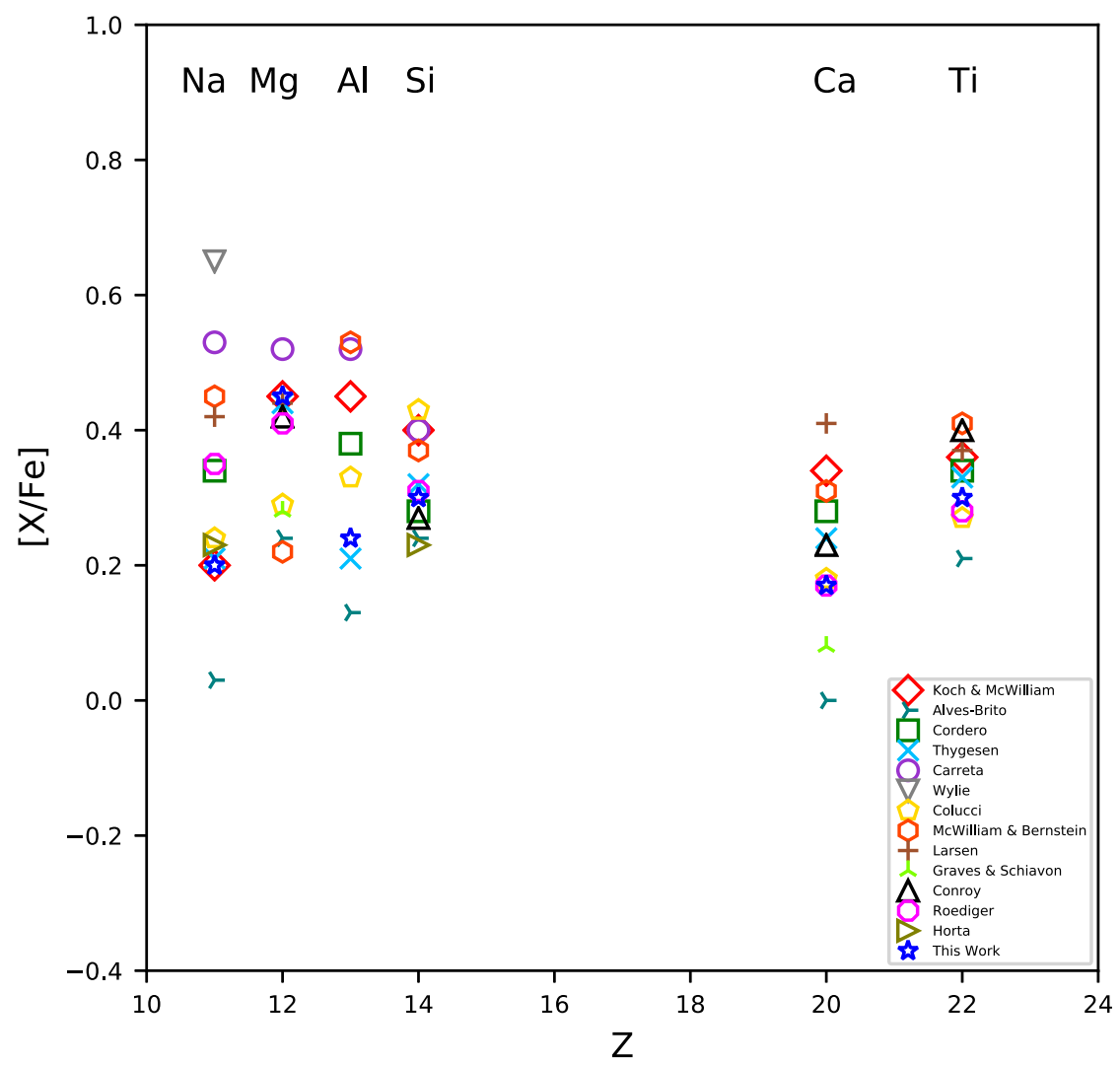

Figure 11. $[\mathrm{X} / \mathrm{Fe}]$ versus $\mathrm{Z}$ for present results and previous literature results.

et al. (2017) have clearly demonstrated that essentially all globular clusters show a first (1G) and a second generation (2G). For 47 Tuc, Milone et al. (2017) found a ratio of first generation to total number of stars of $N_{1} / N_{\text {TOT }}=0.175$. Helium abundances are expected to be somewhat enhanced in 2G stars (Lagioia et al. 2018; Milone et al. 2018), but for the helium small enhancements measured, there is essentially no impact on the building of integrated spectra (see for example Fig. 2 by Souza et al. 2020).

We here try to identify the effect of MPs in integrated spectra by measuring the $\mathrm{Na}$ abundance. $\mathrm{Na}$ is a major indicator of MPs, with second generation stars showing strong $\mathrm{Na}$ abundances, as clearly demonstrated in Carretta et al. (2009, 2019). In the integrated spectra, the presence of MPs would be seen in an overall enhanced $\mathrm{Na}$ abundance. In order to check this possibility, in Fig. 10, the synthetic spectra are computed for $[\mathrm{Na} / \mathrm{Fe}]=0.0,+0.3$ and the best-fitting abundances are reported in Table 4 line-by-line. Na I $8194.79 \AA$ line shows an Na deficiency, lines Na I 5682.63, 6160.75 and $8183.25 \AA$ show a moderate enhancement and only lines NaI 5688.20 and $6154.23 \AA$ indicate a strong enhancement. From this, we conclude that the Na abundance is not a clear indicator of MPs for 47 Tuc with the present integrated spectra data. A higher resolution integrated spectrum would be needed to obtain a more precise measurement.

\subsection{Associated errors}

The resulting mean abundances derived for $\mathrm{Mg}, \mathrm{Al}, \mathrm{Si}, \mathrm{Ca}, \mathrm{Ti}, \mathrm{Ba}$, and Eu are summarized in Table 5. The errors were estimated as the standard error of the mean, i.e. $[\mathrm{X} / \mathrm{Fe}]_{\mathrm{error}}=\sigma_{[\mathrm{X} / \mathrm{Fe}]} / \sqrt{N_{\mathrm{X}}-1}$, where $\sigma_{[\mathrm{X} / \mathrm{Fe}]}$ is the standard deviation of the abundances derived from different spectral lines and $N_{\mathrm{X}}$ in the number of lines used.
Since the Eu abundance was estimated from a single Eu I line, no uncertainty is quoted for this element in Table 5.

The choice of age within the literature ages, has very little effect on the resulting spectra and consequently also on the abundance ratios as shown in Moura et al. (2019). The choice of metallicity within the values form the literature and of $[\mathrm{alpha} / \mathrm{Fe}]=+0.4$ also has little impact on the abundance ratios, given that the possible small differences in the models are cancelled out.

The variance in abundance from the different lines is due to noise in the observed spectra, blends, uncertainties on oscillator strengths, and/or on damping constants in the case of strong lines and fit to the continuum. Such variations are found as well in the analysis of individual stars at high spectral resolution, as can be seen e.g. in Alves-Brito et al. (2005), Thygesen et al. (2014), and other analyses of 47 Tuc (Table 1).

\subsection{Comparison with literature}

Fig. 11 compares the present abundance results with literature abundances reported in Table 2, showing that the abundances derived show good agreement with the previous literature. Si and Ti abundances show the smallest spread among authors. Ca shows a small spread among most literature results. Alves-Brito et al. (2005) results, based on high-resolution spectra gives the lowest abundances of $\mathrm{Na}, \mathrm{Al}, \mathrm{Si}, \mathrm{Ca}$, and $\mathrm{Ti}$, increasing the spread for these elements. $\mathrm{Na}$ instead shows the largest spread, which can be explained by a multiple stellar population effect, combined with spread among the studied lines, as shown in Fig. 10. 
Table 4. List of lines inspected in the integrated spectra and resulting abundances.

\begin{tabular}{|c|c|c|c|c|c|}
\hline Species & $\lambda(\AA)$ & $\chi_{\text {ex }}(\mathrm{eV})$ & $\log g f$ & $\mathrm{C}_{6}$ & {$[\mathrm{X} / \mathrm{Fe}]_{47 \mathrm{Tuc}}$} \\
\hline $\mathrm{Na} I$ & 5682.633 & 2.10 & -0.71 & $0.338 \mathrm{E}-29$ & +0.10 \\
\hline $\mathrm{Na} I$ & 5688.205 & 2.11 & -0.45 & $0.538 \mathrm{E}-29$ & +0.60 \\
\hline $\mathrm{Na} \mathrm{I}$ & 6154.230 & 2.10 & -1.56 & $0.900 \mathrm{E}-31$ & +0.35 \\
\hline $\mathrm{Na} I$ & 6160.753 & 2.10 & -1.26 & $0.300 \mathrm{E}-30$ & +0.15 \\
\hline $\mathrm{Na} I$ & 8183.256 & 2.10 & -0.47 & $0.350 \mathrm{E}-30$ & +0.15 \\
\hline $\mathrm{Na} I$ & 8194.790 & 2.10 & +0.24 & $0.350 \mathrm{E}-30$ & -0.15 \\
\hline $\mathrm{Mg}_{\mathrm{I}}$ & 5167.321 & 2.70 & -0.854 & $0.300 \mathrm{E}-29$ & +0.45 \\
\hline $\mathrm{Mg}_{\mathrm{I}}$ & 5172.684 & 2.70 & -0.363 & $0.300 \mathrm{E}-29$ & +0.45 \\
\hline $\mathrm{Mg}_{\mathrm{I}}$ & 5183.604 & 2.70 & -0.168 & $0.300 \mathrm{E}-29$ & +0.45 \\
\hline $\mathrm{Mg}_{\mathrm{I}}$ & 5528.405 & 4.34 & -0.547 & $0.163 \mathrm{E}-29$ & +0.46 \\
\hline $\mathrm{Mg}_{\mathrm{I}}$ & 8806.756 & 4.34 & -0.144 & $0.123 \mathrm{E}-30$ & +0.44 \\
\hline $\mathrm{Al} \mathrm{I}$ & 6696.185 & 4.02 & -1.58 & $0.300 \mathrm{E}-31$ & +0.24 \\
\hline Al I & 6696.204 & 4.02 & -1.58 & $0.300 \mathrm{E}-31$ & +0.24 \\
\hline Al I & 6696.788 & 4.02 & -1.42 & $0.300 \mathrm{E}-31$ & +0.24 \\
\hline $\mathrm{Al}$ I & 6698.673 & 3.14 & -1.65 & $0.300 \mathrm{E}-31$ & +0.24 \\
\hline Si I & 5948.545 & 5.08 & -1.23 & $0.219 \mathrm{E}-29$ & +0.42 \\
\hline Si I & 7405.772 & 5.61 & -0.66 & $0.182 \mathrm{E}-29$ & +0.32 \\
\hline Si I & 7415.948 & 5.61 & -0.73 & $0.182 \mathrm{E}-29$ & +0.32 \\
\hline Si I & 7423.496 & 5.62 & -0.58 & $0.184 \mathrm{E}-29$ & +0.12 \\
\hline $\mathrm{Ca} I$ & 6102.723 & 1.88 & -0.79 & $0.200 \mathrm{E}-29$ & +0.24 \\
\hline $\mathrm{CaI}$ & 6122.217 & 1.89 & -0.20 & $0.800 \mathrm{E}-29$ & +0.29 \\
\hline $\mathrm{Ca} \mathrm{I}$ & 6161.295 & 2.51 & -1.02 & $0.598 \mathrm{E}-30$ & +0.36 \\
\hline $\mathrm{CaI}$ & 6162.167 & 1.89 & -0.09 & $0.454 \mathrm{E}-30$ & +0.36 \\
\hline $\mathrm{CaI}$ & 6166.440 & 2.52 & -0.90 & $0.595 \mathrm{E}-30$ & +0.24 \\
\hline $\mathrm{Ca} \mathrm{I}$ & 6169.044 & 2.52 & -0.54 & $0.595 \mathrm{E}-30$ & +0.24 \\
\hline $\mathrm{CaI}$ & 6169.564 & 2.52 & -0.27 & $0.598 \mathrm{E}-30$ & +0.24 \\
\hline $\mathrm{CaI}$ & 6439.080 & 2.52 & +0.30 & $0.512 \mathrm{E}-31$ & +0.02 \\
\hline $\mathrm{CaI}$ & 6455.605 & 2.52 & -1.35 & $0.509 \mathrm{E}-31$ & +0.15 \\
\hline $\mathrm{Ca} I$ & 6717.687 & 2.71 & -0.61 & $0.619 \mathrm{E}-30$ & +0.29 \\
\hline $\mathrm{Ca} I$ & 8498.023 & 1.69 & -1.312 & $0.900 \mathrm{E}-32$ & +0.24 \\
\hline $\mathrm{CaI}$ & 8542.091 & 1.70 & -0.362 & $0.800 \mathrm{E}-32$ & +0.24 \\
\hline $\mathrm{Ca} I$ & 8662.141 & 1.69 & -0.623 & $0.800 \mathrm{E}-32$ & +0.24 \\
\hline Ti I & 5866.449 & 1.07 & -0.84 & $0.216 \mathrm{E}-31$ & +0.10 \\
\hline Ti I & 5922.108 & 1.05 & -1.46 & $0.346 \mathrm{E}-31$ & +0.33 \\
\hline Ti I & 5941.750 & 1.05 & -1.53 & $0.346 \mathrm{E}-31$ & +0.44 \\
\hline Ti I & 5965.825 & 1.88 & -0.42 & $0.214 \mathrm{E}-31$ & +0.34 \\
\hline Ti I & 5978.539 & 1.87 & -0.53 & $0.214 \mathrm{E}-31$ & +0.33 \\
\hline Ti I & 6126.214 & 1.07 & -1.43 & $0.206 \mathrm{E}-31$ & +0.32 \\
\hline Ti I & 6261.106 & 1.43 & -0.48 & $0.468 \mathrm{E}-31$ & +0.33 \\
\hline Ti I & 6266.010 & 1.75 & -2.98 & $0.300 \mathrm{E}-31$ & +0.33 \\
\hline Ti I & 6556.077 & 1.46 & -1.07 & $0.274 \mathrm{E}-31$ & +0.32 \\
\hline Ti I & 6743.127 & 0.90 & -1.73 & $0.289 \mathrm{E}-31$ & +0.20 \\
\hline Ti II & 5154.068 & 1.57 & -1.75 & $0.200 \mathrm{E}-29$ & +0.32 \\
\hline Ti II & 5336.771 & 1.58 & -1.70 & $0.900 \mathrm{E}-32$ & +0.37 \\
\hline Ti II & 5381.021 & 1.57 & -2.08 & $0.890 \mathrm{E}-32$ & +0.37 \\
\hline Ti II & 5418.751 & 1.58 & -2.13 & $0.300 \mathrm{E}-30$ & +0.32 \\
\hline Ba II & 5853.675 & 0.60 & -1.10 & $0.300 \mathrm{E}-31$ & +0.36 \\
\hline Ba II & 6141.713 & 0.70 & -0.08 & $0.300 \mathrm{E}-31$ & +0.32 \\
\hline Ba II & 6496.90 & 0.60 & -0.32 & $0.300 \mathrm{E}-31$ & +0.32 \\
\hline Eu II & 6645.064 & 1.38 & +0.12 & $0.120 \mathrm{E}-31$ & +0.32 \\
\hline
\end{tabular}

\section{SUMMARY}

47 Tuc is a reference moderately metal-rich globular cluster and has been extensively studied and used as template for other fainter objects. Our aim is to show that the integrated spectra of globular clusters is a useful tool to derive element abundances. In this work, we show that the SYNSSP package suitably reproduces the WAGGS observed integrated spectra of $47 \mathrm{Tuc}$ and in particular that the prominent lines of $\mathrm{Na}, \mathrm{Mg}, \mathrm{Si}, \mathrm{Ca}, \mathrm{Ti}, \mathrm{Ba}$, and $\mathrm{Eu}$ can be used to derive abundances. Together with the results obtained in Moura et al. (2019), this work indicates that the employed techniques are a step towards using IL together with synthetic spectra for the analysis of more complex and distant objects, such as globular clusters in external galaxies.

The abundances calculated for 47 Tuc show good agreement with the previous literature, including results from HRS of individual stars. Ti abundances are the best matched from all methods. Na instead shows the largest spread and this is probably due to the multiple stellar population effects, combined with spread among the studied lines (Section 5.7). The wings of strong lines are well-fitted, based on the abundances derived from the weaker lines, which can be useful for a future more precise calibration of well-known indices such as $\mathrm{Mg}_{2}$ and CaT lines. 


\section{ACKNOWLEDGEMENTS}

CR acknowledges a CAPES Master fellowship. BB acknowledges grants from CAPES - Financial code 001, CNPq, and FAPESP. TM acknowledges the FAPESP postdoctoral fellowship no. 2018/034807. MT thanks the support of CNPq (process \#307675/2018-1) and the program L'Oréal UNESCO ABC Para Mulheres na Ciência.

\section{DATA AVAILABILITY}

The observed data analysed in this work are available in the literature. The computed data underlying this article will be shared on reasonable request to the corresponding author.

\section{REFERENCES}

Alvarez R., Plez B., 1998, A\&A, 330, 1109

Alves-Brito A. et al., 2005, A\&A, 435, 657

Anderson J. et al., 2008, AJ, 135, 2055

Anstee S. D., O’Mara B. J., 1995, MNRAS, 276, 859

Armandroff T. E., 1989, AJ, 97, 375

Barbuy B., Chiappini C., Gerhard O., 2018b, ARA\&A, 56, 223

Barbuy B., Trevisan J., de Almeida A., 2018a, PASA, 35, 46

Barklem P. S., O’Mara B. J., 1997, MNRAS, 290, 102

Barklem P. S., O’Mara B. J., Ross J. E., 1998, MNRAS, 296, 1057

Baumgardt H., Hilker M., Sollima A., Bellini A., 2019, MNRAS, 482, 5138

Bedin L., Piotto G., Anderson J., Cassisi S., King I. R., Momany Y., Carraro G., 2004, ApJ, 605, L125

Bica E., Alloin D., Schmidt A., 1990, MNRAS, 242, 241

Bica E., Bonatto C., Barbuy B., Ortolani S., 2006, A\&A, 450, 105

Bica E., Ortolani S., Barbuy B., 2016, PASA, 33, e28

Brogaard K., VandenBerg D. A., Bedin L. R., Milone A. P., Thygesen A., Grundahl F., 2017, MNRAS, 468, 645B

Burstein D., Faber S., Gaskell C., Krumm N., 1984, ApJ, 287, 586

Carretta E., 2019, A\&A, 624, A24

Carretta E., Bargaglia A., Gratton R., Bonifacio P., Pasquini L., 2004, A\&A, 416, 925

Carretta E., Bargaglia A., Gratton R., D’Orazi V., Lucatello S., 2009, A\&A, 508, 695

Carretta E., Bragaglia A., Gratton R., Lucatello S., 2009, A\&A, 505, 139

Castelli F., Kurucz R. L., 2004, in Piskunov N., Weiss W. W., Gray D. F., eds, IAU Symp. 210, Modelling of Stellar Atmospheres. Astron. Soc. Pac., San Francisco, p. A20

Colucci J., Bernstein R., McWilliam A., 2017, ApJ, 834, 105

Conroy C., Villaume A., van Dokkum P., Lind K., 2018, ApJ, 854, 139

Cordero M. J., Pilachowski C. A., Johnson C. I., McDonald I., Zijlstra A. A., Simmerer J., 2014, ApJ, 780, 94

Cordier D., Pietrinferni A., Cassisi S., Salaris M., 2007, AJ, 133, 468

Denissenkov P. A., VandenBerg D. A., Kopacki G., Ferguson J. W., 2017, ApJ, 849, 159D

Dickens R. J., Bell R. A., Gustafsson B., 1979, ApJ, 232, 428

Dotter A., Chaboyer B., Jevremovic D., Kostov V., Baron E., Ferguson J.W., 2008, ApJS, 178, 89

Dotter A., Kaluzny J., Thompson I., 2009, IAUS, 258, 171

Gaia Collaboration et al., 2018a, A\&A, 616, A1

Gaia Collaboration et al., 2018b, A\&A, 616, A12

Girardi L., Bressan A., Bertelli G., Chiosi C., 2000, A\&AS, 141, 371

Gratton R., Sneden C., Carretta E., 2004, ARA\&A, 42, 385

Gratton R. G., Bragaglia A., Carretta E., 2003, A\&A, 408, 529

Gratton R. G., Carretta E., Bragaglia A., 2012, A\&ARv, 20, 50

Graves G., Schiavon R., 2008, ApJS, 177, 446

Gustafsson B., Edvarsson B., Eriksson K., Jørgensen U. G., Nordlund Å., Plez B., 2008, A\&A, 486, 951
Hansen B. M. S. et al., 2013, Nature, 500, 51

Harris W. E., 1996, AJ, 112, 1487 (edition of 2010)

Horta D. et al., 2020, MNRAS, 493, 3363

Koch A., McWilliam A., 2008, AJ, 135, 1551

Kroupa P., 2002, Science, 295, 82

La Barbera F., Ferreras I, Vazdekis A., de la Rosa I.G., de Carvalho R.R., Trevisan M, Falcón-Barroso J., Ricciardelli E., 2013, MNRAS, 433, 3017 Lagioia E. P. et al., 2018, MNRAS, 475, 4088

Lapenna E., Mucciarelli A., Lanzoni B., Ferraro F. R., Dalessandro E., Origlia L., Massari D., 2014, ApJ, 797, 124

Larsen S., Brodie J., Strader J., 2017, A\&A, 601, A96

Lee Y.-W., Joo J. M., Sohn Y. J., 1999, Nature, 402, 55

Majewski S. R., Schiavon R. P., Frinchaboy P. M., 2017, AJ, 154, 94

Mann A. W., Feiden G. A., Gaidos E., Boyajian T., von Braun K., 2015, ApJ, 804, 64

McWilliam A., 2016, PASA, 33, 40

McWilliam A., Bernstein R., 2008, ApJ, 684, 326

McWilliam A., Preston G. W., Sneden C., Shectman S., 1995, AJ, 109, 2736

Milone A. P. et al., 2017, MNRAS, 469, 800

Milone A. P. et al., 2018, MNRAS, 481, 5098

Moura T., Trevisan M., Barbuy B., Rossi S., 2019, ApJ, 885, 28

Norris J., Cottrell P., 1979, ApJ, 229, L69

Ortolani S., Renzini A., Gilmozzi R., Marconi G., Barbuy B., Bica E., Rich R. M., 1995, Nature, 377, 701

Osborn W., 1971, Obs., 91, 223

Pancino E., Bellazzini M., Giuffrida G., Marinoni S., 2017, MNRAS, 467, 412

Pehlivan Rhodin A., Hartman H., Nilsson H., Jönsson P., 2017, A\&A, 598, A102

Pérez-Villegas A., Barbuy B., Kerber L.O., Ortolani S., Souza S.O., Bica E., 2020, MNRAS, 491, 3251

Pietrinferni A., Cassisi S., Salaris M., Castelli F., 2004, ApJ, 612, 168

Pietrinferni A., Cassisi S., Salaris M., Castelli F., 2006, ApJ, 642, 797

Pilachowski C. A., Canterna R., Wallerstein G., 1980, ApJ, 235, L21

Piotto G. et al., 2015, AJ, 149, 91

Plez B., 2012, Astrophysics Source Code Library, record ascl:1205.004

Prugniel P., Vauglin I., Koleva M., 2011, A\&A, 531, A165

Roediger J., Corteau S., Graves G., Schiavon R., 2014, ApJS, 210, 10

Salasnich B., Girardi L., Weiss A., Chiosi C., 2000, A\&A, 361, 1023

Salpeter E.E., 1955, ApJ, 121, 161

Sarajedini A. et al., 2007, AJ, 133, 1658

Schiavon R., Faber S. M., Rose J. A., Castilho B. A., 2002, ApJ, 580, 873

Schiavon R., Rose J., Corteau S., MacArthur L., 2005, ApJS, 160, 163

Schiavon R. P., 2007, ApJS, 171, 146

Shapley H., 1918, ApJ, 48, 154

Sharma K., Prugniel P., Singh H. P., 2016, A\&A, 585, A64

Sneden C., 1973, ApJ, 184, 839

Souza S. O., Kerber L., Barbuy B., Pérez-Villegas A., Oliveira R. A. P., Nardiello D., 2020, ApJ, 890, 38

Sánchez-Blázquez P. et al., 2006, MNRAS, 371, 703

Thompson I. B., Kaluzny J., Rucinski S. M., Krzeminski W., Pych W., Dotter A., Burley G. S., 2010, AJ, 139, 329

Thygesen A. et al., 2014, A\&A, 572, A108

Usher C. et al., 2019, MNRAS, 468, 3828

Usher C. et al., 2019, MNRAS, 482, 1275

VandenBerg D. A., Bergsbusch P. A., Ferguson J. W., Edvardsson B., 2014, ApJ, 794, 72V

VandenBerg D. A., Brogaard K., Leaman R., Casagrande L., 2013, ApJ, 775, $134 \mathrm{~V}$

Worthey G., Faber S., Gonzalez J. J., Burstein D., 1994, ApJS, 94, 687

Worthey G., Ottaviani D. L., 1997, ApJS, 111, 377

Wylie E., Cottrell P., Sneden C., Lattanzio J., 2006, ApJ, 649, 248 

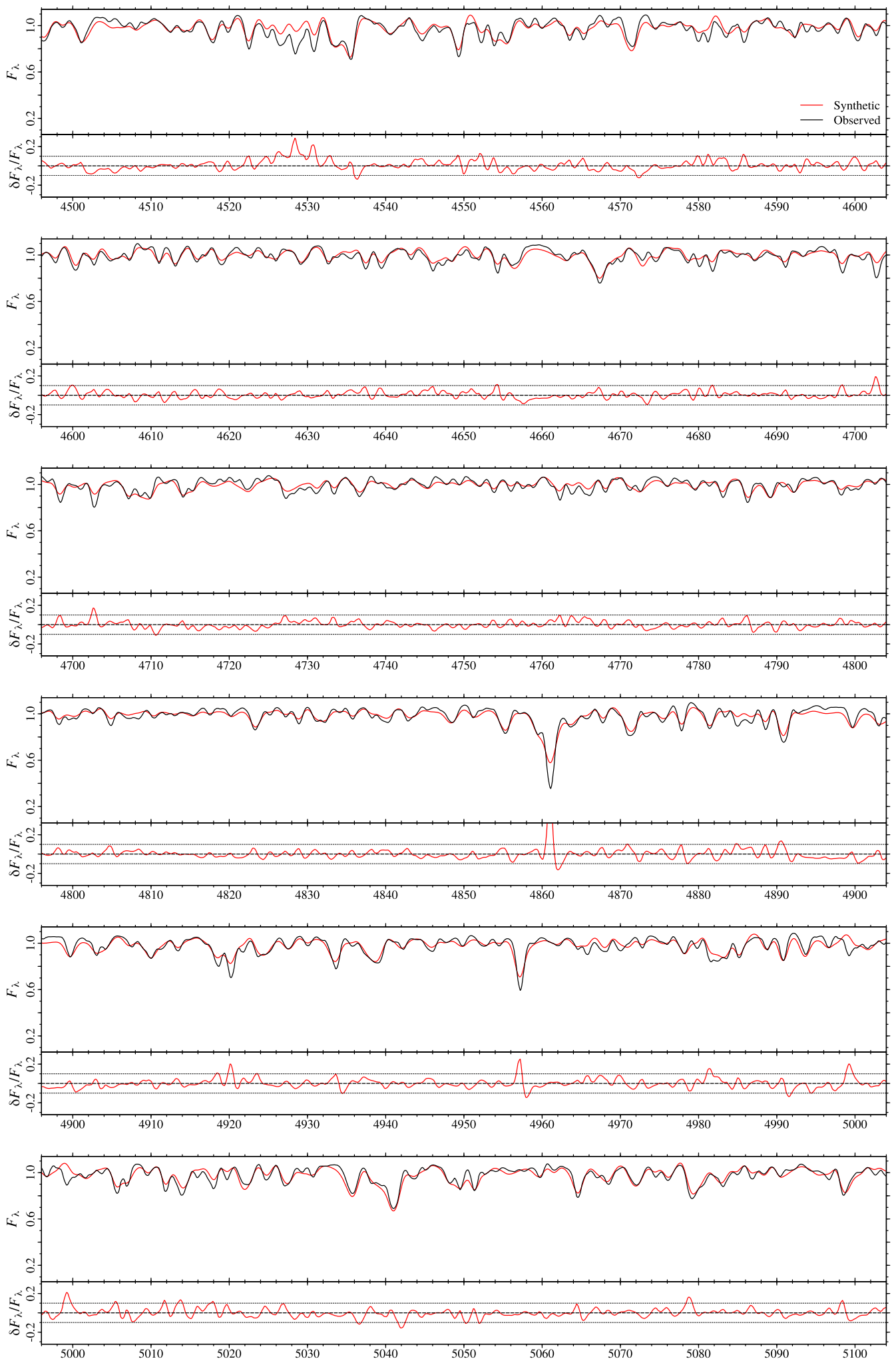

Figure A1. Comparison between synthetic (red) and observed (black) spectra, in the range 4500-5100. 

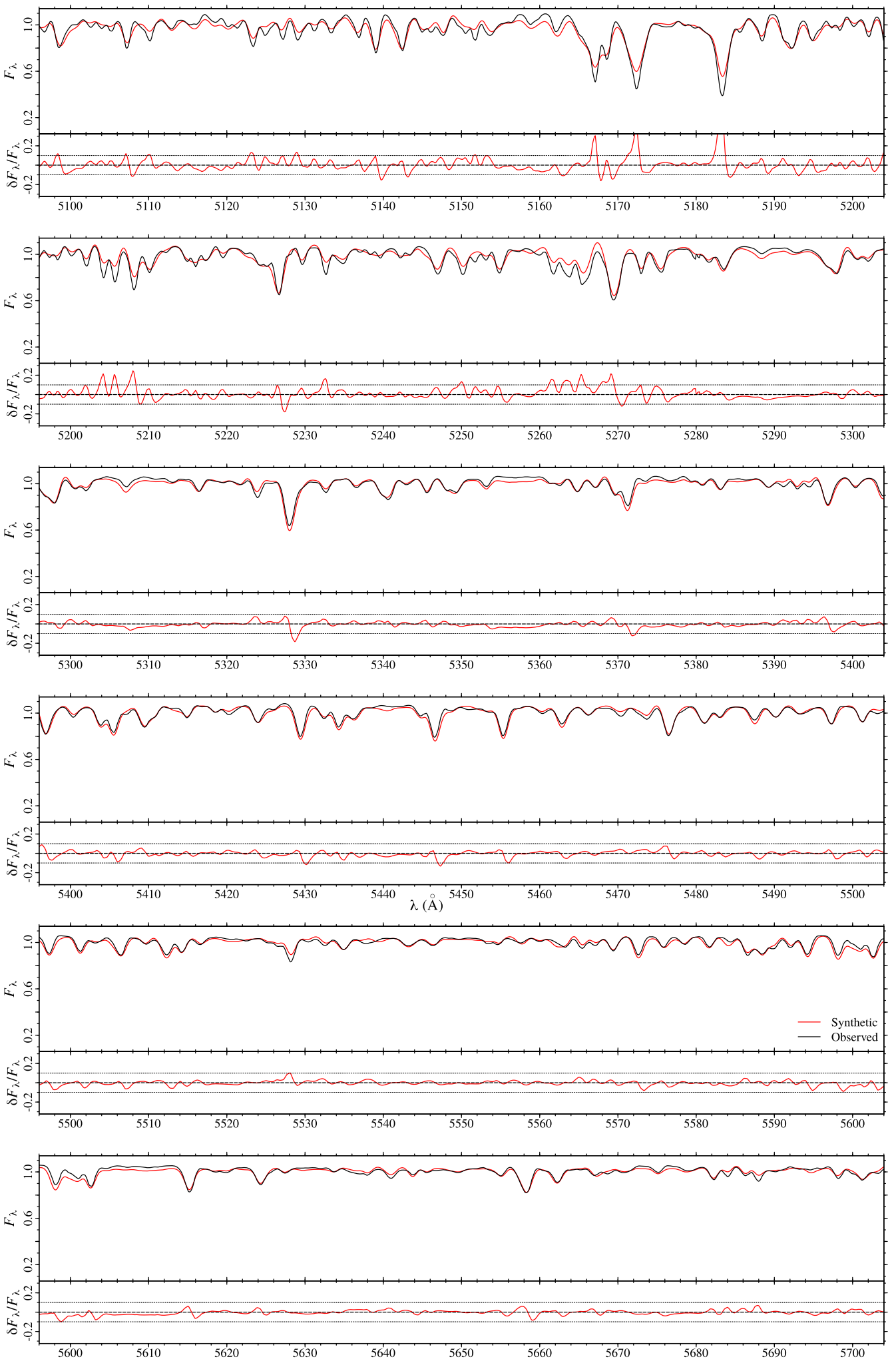

Figure A2. Comparison between synthetic and observed spectra, in the range 5100-5700 ̊. 

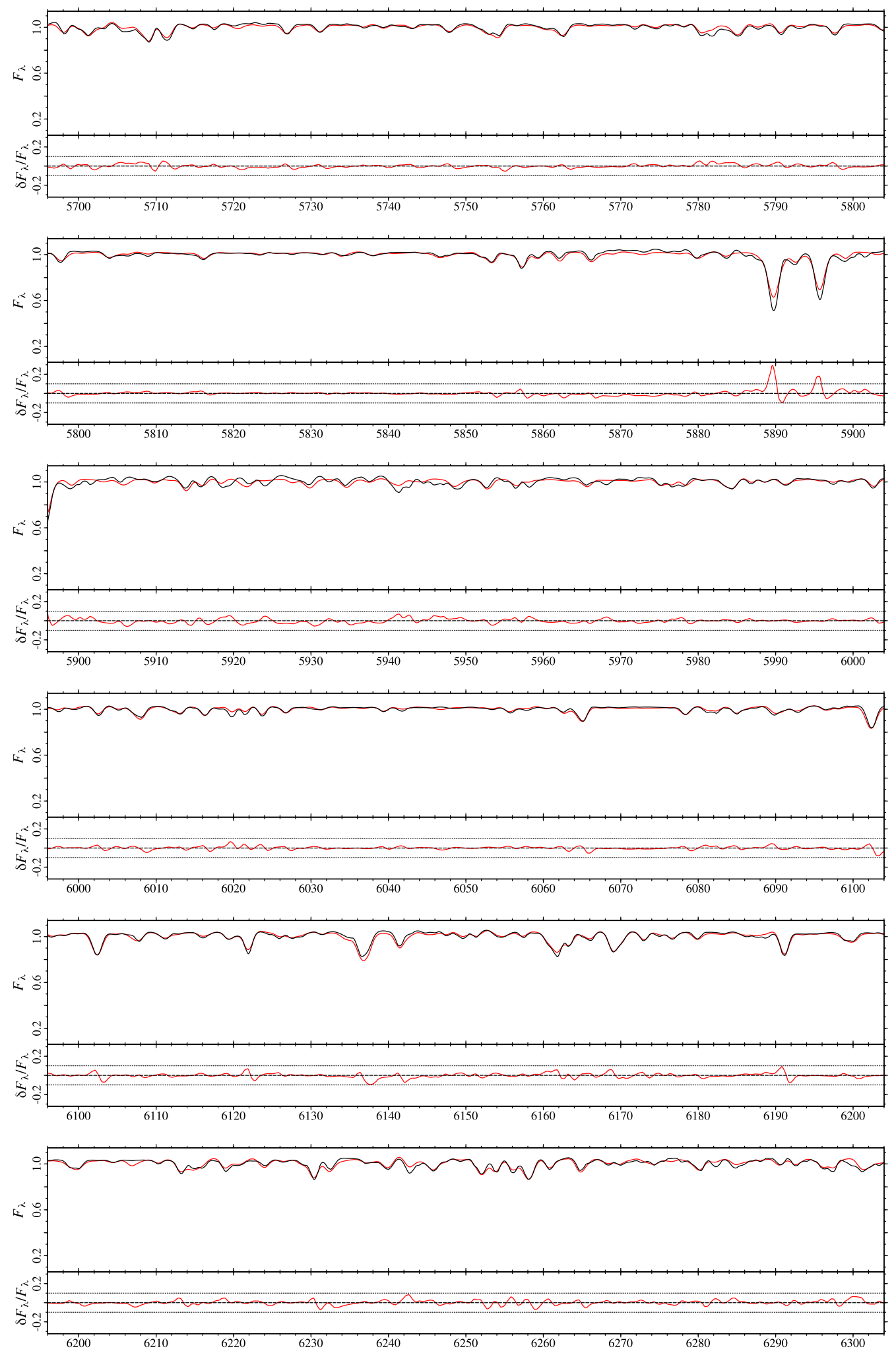

Figure A3. Comparison between synthetic and observed spectra, in the range 5700-6300 ̊. 

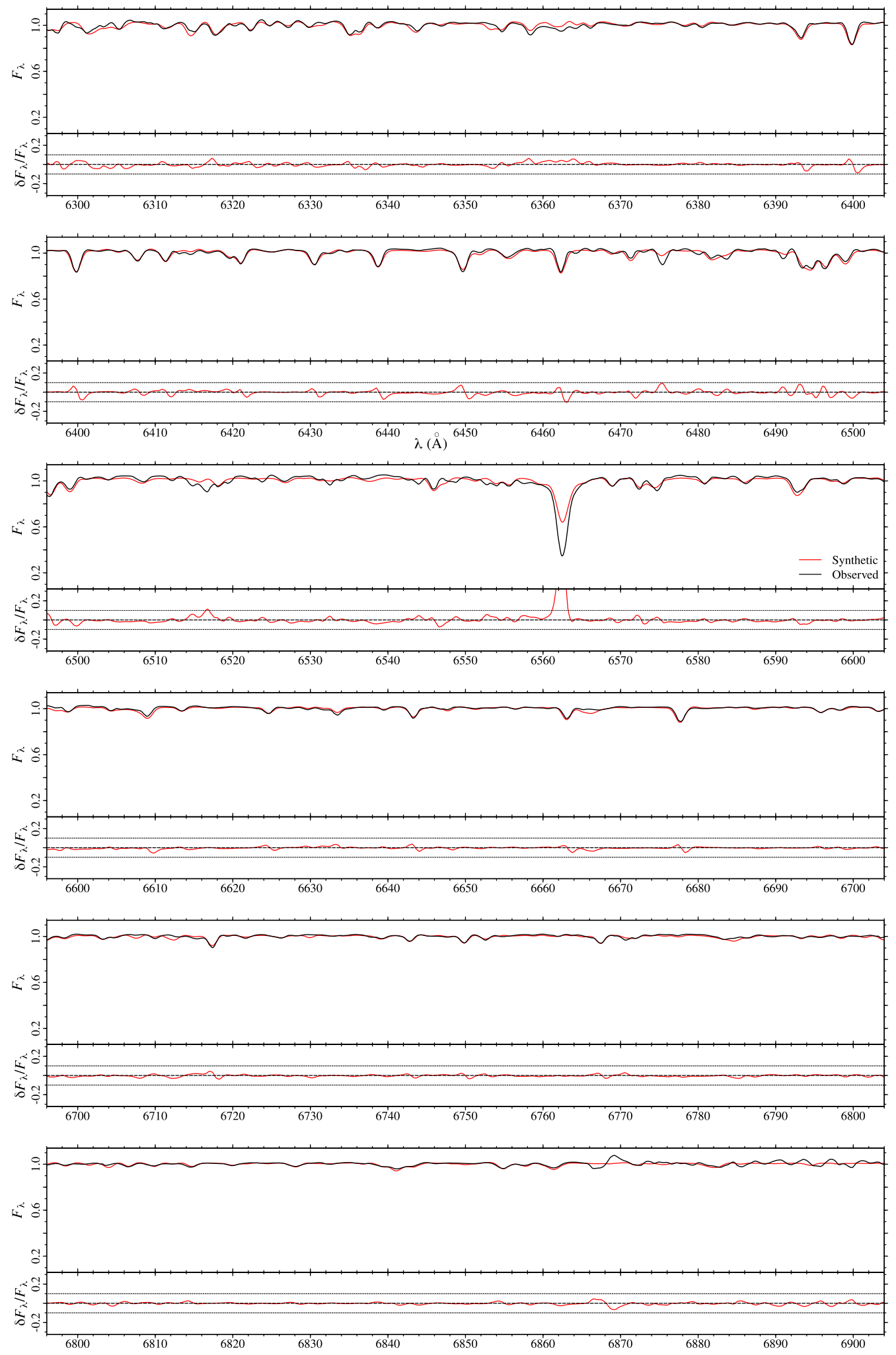

Figure A4. Comparison between synthetic and observed spectra, in the range 6300-6900 ̊. 

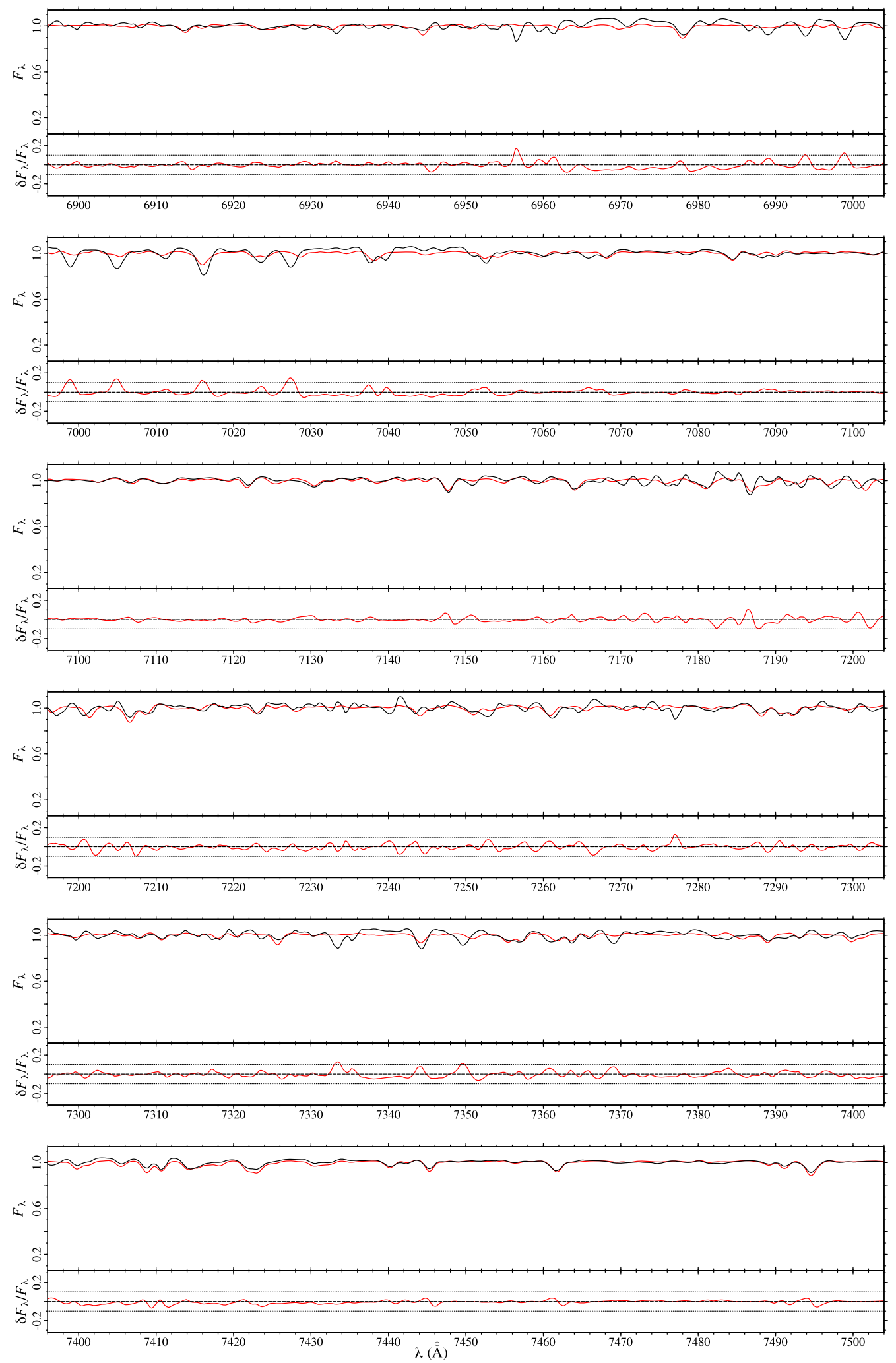

Figure A5. Comparison between synthetic and observed spectra, in the range 6900-7500 ̊. 

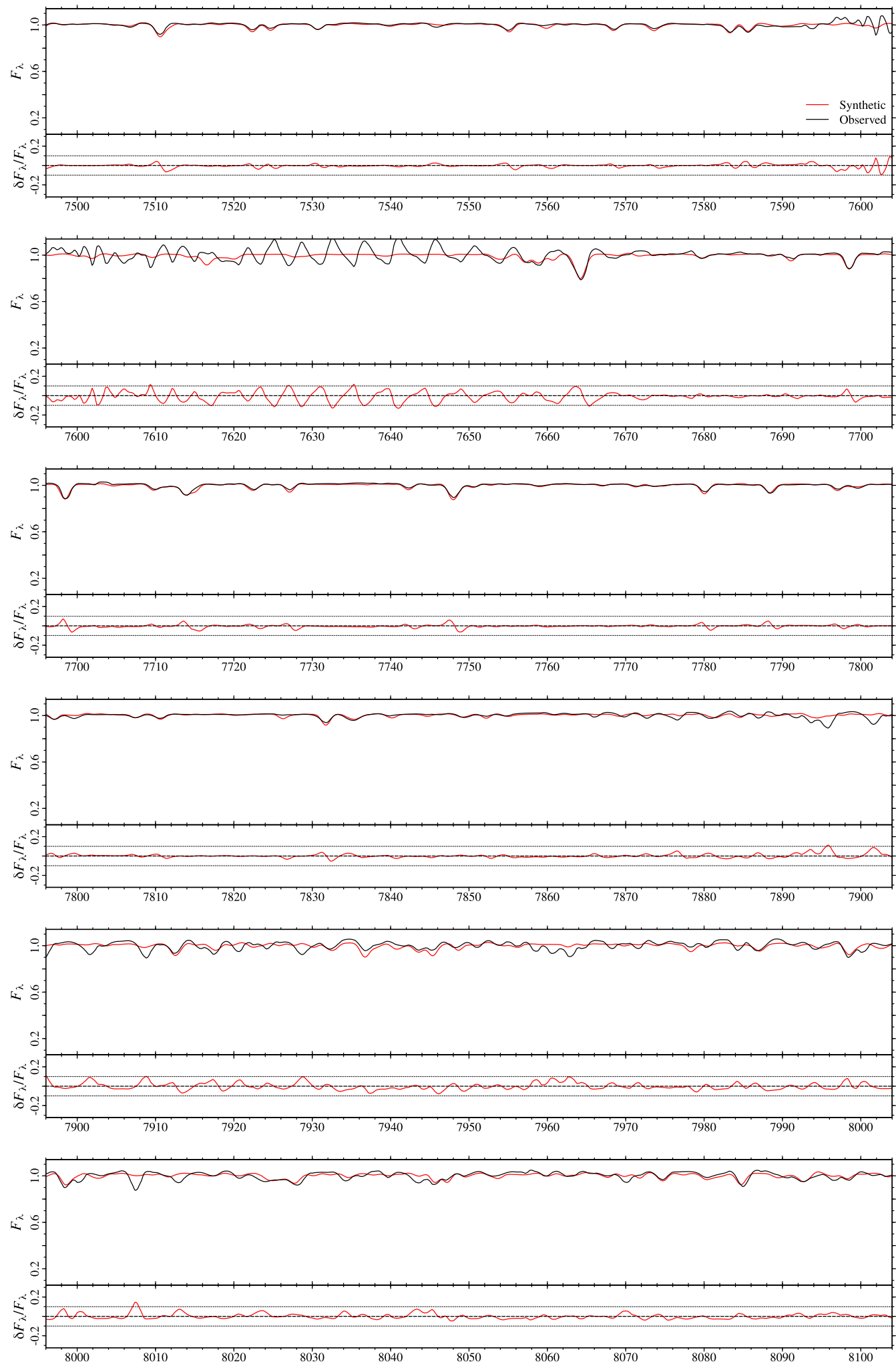

Figure A6. Comparison between synthetic and observed spectra, in the range $7500-8100 \AA$. 

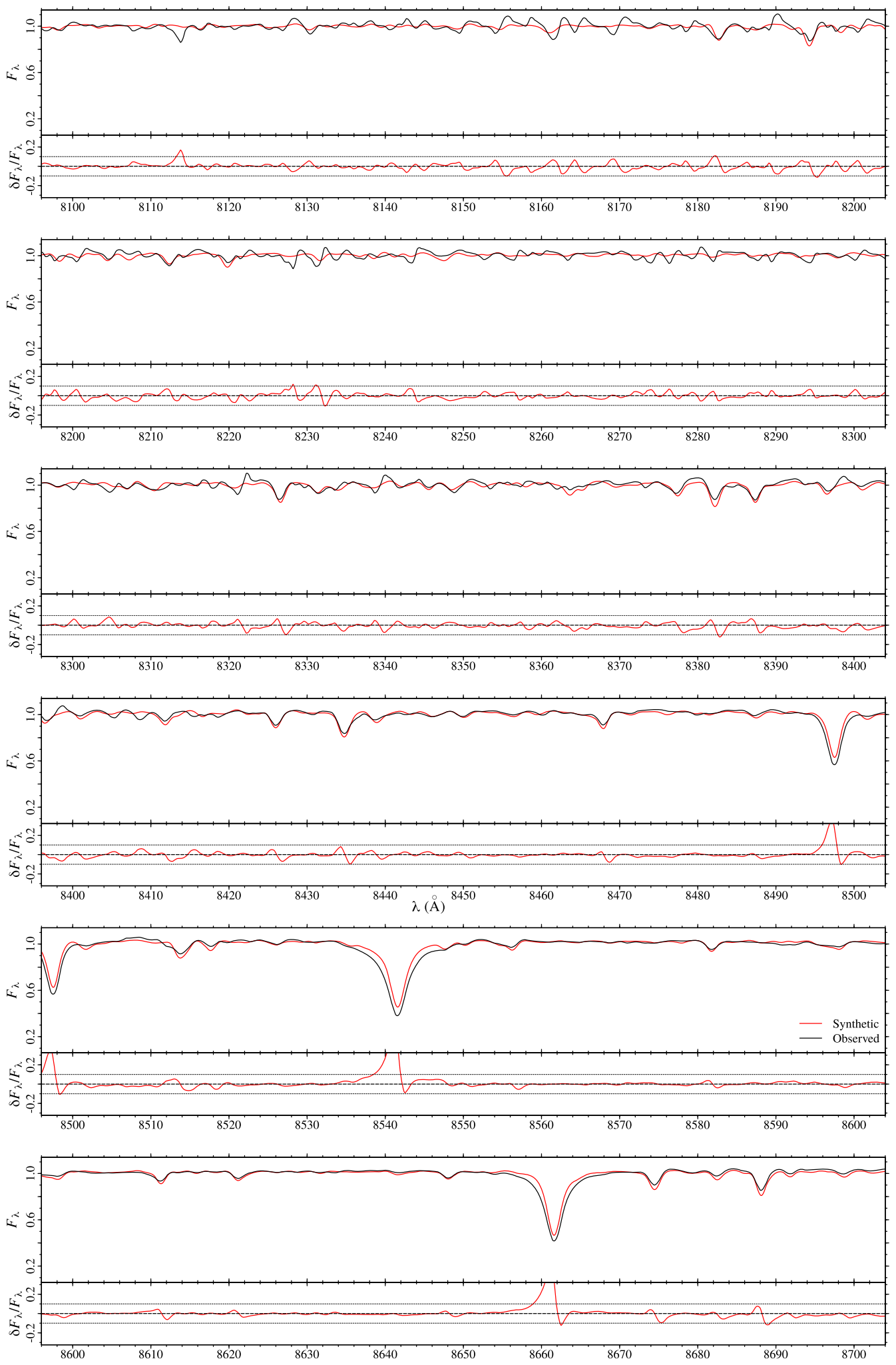

Figure A7. Comparison between synthetic and observed spectra, in the range $8100-8700 \AA$. 

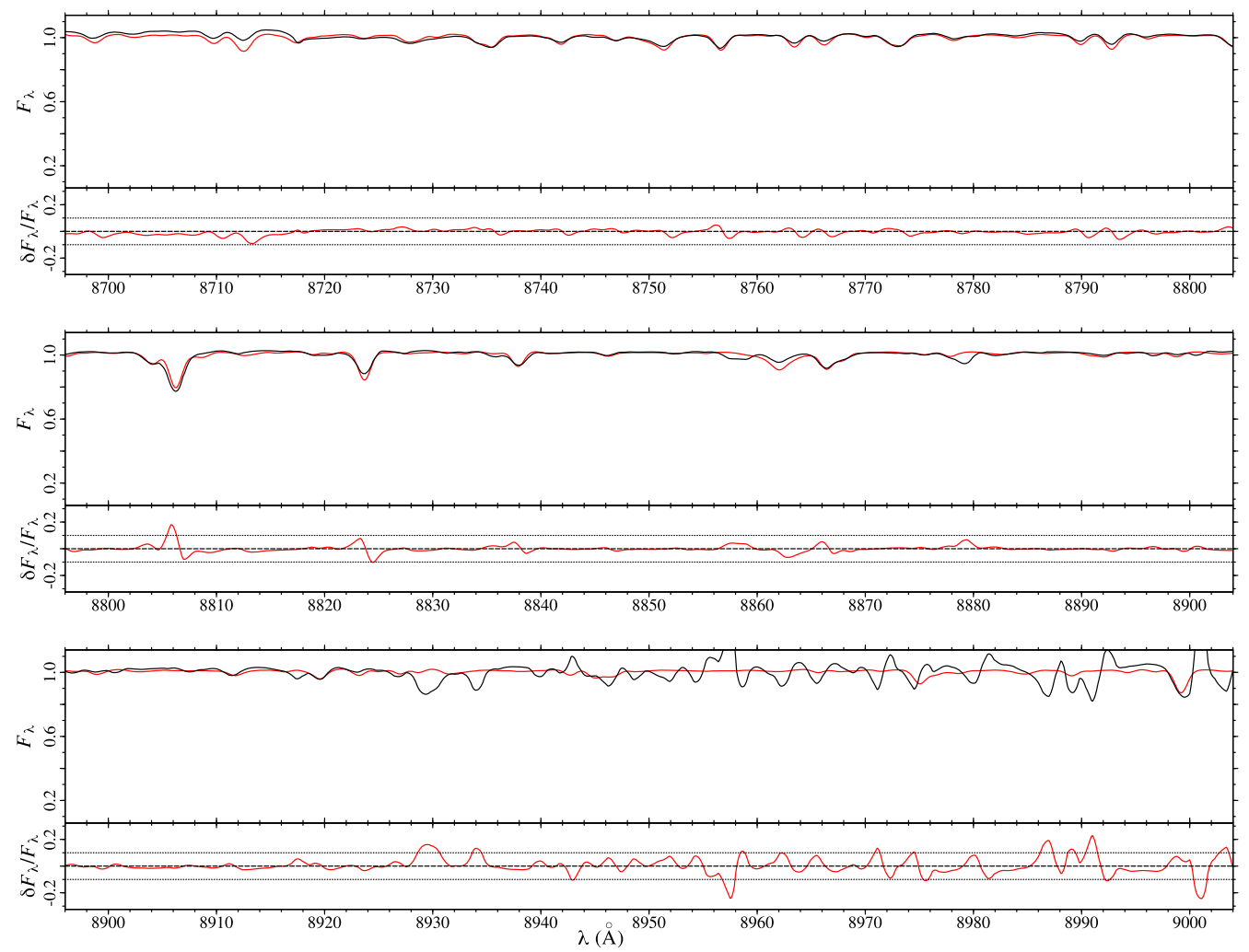

Figure A8. Comparison between synthetic and observed spectra, in the range 8700-9000 ̊.

This paper has been typeset from a $\mathrm{T}_{\mathrm{E}} \mathrm{X} / \mathrm{LT} \mathrm{T} \mathrm{X}$ file prepared by the author. 